\title{
Overview: oxidant and particle photochemical processes above a south-east Asian tropical rainforest (the OP3 project): introduction, rationale, location characteristics and tools
}

C. N. Hewitt ${ }^{1}$, J. D. Lee ${ }^{2}$, A. R. MacKenzie ${ }^{1}$, M. P. Barkley ${ }^{3}$, N. Carslaw ${ }^{4}$, G. D. Carver ${ }^{5}$, N. A. Chappell ${ }^{1}$, H. Coe Co $^{6}$, C. Collier ${ }^{7}$, R. Commane ${ }^{8, *}$, F. Davies ${ }^{7}$, B. Davison ${ }^{1}$, P. DiCarlo ${ }^{9}$, C. F. Di Marco ${ }^{10}$, J. R. Dorsey ${ }^{6}$, P. M. Edwards ${ }^{8}$, M. J. Evans ${ }^{11}$, D. Fowler ${ }^{10}$, K. L. Furneaux ${ }^{* *}$, , M. Gallagher ${ }^{6}$, A. Guenther ${ }^{12}$, D. E. Heard ${ }^{8}$, C. Helfter ${ }^{10}$,

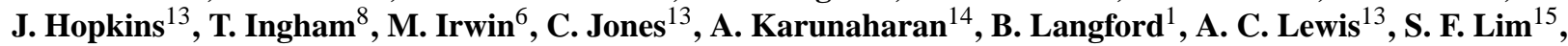
S. M. MacDonald ${ }^{8}$, A. S. Mahajan ${ }^{8}$, S. Malpass ${ }^{4}$, G. McFiggans ${ }^{6}$, G. Mills $^{16}$, P. Misztal ${ }^{10,17}$, S. Moller ${ }^{13}$, P. S. Monks ${ }^{14}$, E. Nemitz ${ }^{10}$, V. Nicolas-Perea ${ }^{14}$, H. Oetjen ${ }^{8}$, D. E. Oram ${ }^{16}$, P. I. Palmer ${ }^{3}$, G. J. Phillips ${ }^{10}$, R. Pike ${ }^{5}$, J. M. C. Plane ${ }^{8}$,

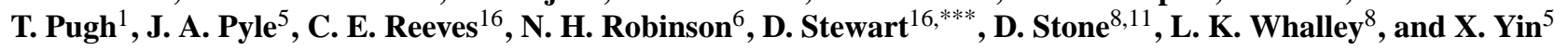

${ }^{1}$ Lancaster Environment Centre, Lancaster University, Lancaster LA1 4YQ, UK

${ }^{2}$ National Centre for Atmospheric Science, University of York, York YO10 5DD, UK

${ }^{3}$ School of GeoSciences, University of Edinburgh, Edinburgh EH9 3JW, UK

${ }^{4}$ Environment Department, University of York, York YO10 5DD, UK

${ }^{5}$ Centre for Atmospheric Science, Department of Chemistry, Cambridge University, Cambridge, CB2 1EW, UK

${ }^{6}$ School of Earth, Atmospheric and Environmental Sciences, University of Manchester, Manchester M13 3PL, UK

${ }^{7}$ Centre for Environmental Systems Research, University of Salford, Salford M5 4WT, UK

${ }^{8}$ School of Chemistry, University of Leeds, Leeds LS2 9JT, UK

${ }^{9}$ CETEMPS - Dipartimento di Fisica, Università di L'Aquila, 67010 Coppito, L'Aquila, Italy

${ }^{10}$ Biogeochemistry Programme, Centre for Ecology and Hydrology, Penicuik, EH26 0QB, UK

${ }^{11}$ School of the Environment, University of Leeds, Leeds, LS2 9JT, UK

${ }^{12}$ National Center for Atmospheric Research, Boulder CO 80301, USA

${ }^{13}$ Department of Chemistry, University of York, York YO10 5DD, UK

${ }^{14}$ Department of Chemistry, University of Leicester, Leicester LE1 7RH, UK

${ }^{15}$ Retired, formerly at Malaysian Meteorological Department, Jalan Sultan, Petaling Jaya, Selangor Darul Ehsan, Malaysia

${ }^{16}$ School of Environmental Sciences, University of East Anglia, Norwich NR4 7TJ, UK

${ }^{17}$ Department of Chemistry, University of Edinburgh, Edinburgh EH9 3JW, UK

* now at: School of Engineering and Applied Sciences, Harvard University, MA, USA

${ }^{* *}$ formerly at: School of Chemistry, University of Leeds, Leeds LS2 9JT, UK

${ }^{* * *}$ now at: Department of Chemistry, University of Reading, Reading RG6 6AH, UK

$\dagger$ deceased

Received: 8 July 2009 - Published in Atmos. Chem. Phys. Discuss.: 11 September 2009

Revised: 4 December 2009 - Accepted: 9 December 2009 - Published: 12 January 2010

Abstract. In April-July 2008, intensive measurements were made of atmospheric composition and chemistry in Sabah, Malaysia, as part of the "Oxidant and particle photochemical processes above a South-East Asian tropical rainfor-

Correspondence to: C. N. Hewitt (n.hewitt@lancaster.ac.uk) est" (OP3) project. Fluxes and concentrations of trace gases and particles were made from and above the rainforest canopy at the Bukit Atur Global Atmosphere Watch station and at the nearby Sabahmas oil palm plantation, using both ground-based and airborne measurements. Here, the measurement and modelling strategies used, the characteristics of the sites and an overview of data obtained are described. Composition measurements show that the rainforest 
site was not significantly impacted by anthropogenic pollution, and this is confirmed by satellite retrievals of $\mathrm{NO}_{2}$ and HCHO. The dominant modulators of atmospheric chemistry at the rainforest site were therefore emissions of BVOCs and soil emissions of reactive nitrogen oxides. At the observed BVOC: $\mathrm{NO}_{\mathrm{x}}$ volume mixing ratio ( $\left.\sim 100 \mathrm{pptv} / \mathrm{pptv}\right)$, current chemical models suggest that daytime maximum $\mathrm{OH}$ concentrations should be ca. $10^{5}$ radicals $\mathrm{cm}^{-3}$, but observed $\mathrm{OH}$ concentrations were an order of magnitude greater than this. We confirm, therefore, previous measurements that suggest that an unexplained source of $\mathrm{OH}$ must exist above tropical rainforest and we continue to interrogate the data to find explanations for this.

\section{Introduction}

Tropical and equatorial forests account for over half of the World's forests ( 1.8 billion ha) and act as a massive source of matter and energy to the lower atmosphere. They exhibit some of the most dynamic yet poorly understood biogeochemical behaviour on Earth. This behaviour is driven by solar radiation and is largely mediated by its transformation into latent and sensible heat, with the concomitant uptake of carbon by photosynthesis and the associated emission of reactive, less-reactive and un-reactive trace gases, water vapour and energy into the atmosphere. Simultaneously, ozone and other trace gases, aerosol particles, and momentum, are lost to the forest surface. A further important consequence of the large solar radiation flux in the tropics is the very vigorous convective uplift that occurs, which results in the rapid movement of chemical species emitted at or near ground level into the free troposphere, as shown, for example, in Surinam (Andreae et al., 2001). Hence reactive trace gas emissions from the surface in the tropics may take part in chemical processes at greater distances and at higher altitudes from their sources than might otherwise occur.

Globally, tropical and equatorial forests are estimated to account for almost half of all biogenic reactive volatile organic compound (VOC) emissions into the atmosphere (Guenther et al., 1995, 2006: global total $1150 \mathrm{Tg}$ C/y, estimate for tropical forests $\sim 500 \mathrm{Tg} \mathrm{C} / \mathrm{y}$ ). These compounds are believed to play a major role in mediating the chemistry of the atmosphere, yet their roles in controlling chemical budgets and processes in the atmosphere on the local, regional and global scales are poorly understood, with considerable and surprising gaps and uncertainties in knowledge remaining (e.g. Lelieveld et al., 2008). In addition, it is possible that biological primary and secondary organic particles play a pivotal role in the formation of cloud condensation nuclei $(\mathrm{CNN})$ and thus control precipitation patterns in forested regions (Barth et al., 2005).

Most previous work on the interactions between tropical forests and atmospheric composition has been carried out in
Amazonia (e.g. the LBA project: Andreae et al., 2002; Avissar et al., 2002), with less in Africa (e.g. the AMMA project: Redelsperger et al., 2006) and very little in SE Asia. Unlike the LBA and AMMA domains, which are contiguous continental regions, the complex mosaic of tropical seas and islands that exists in SE Asia makes the likely atmospheric chemistry occurring there somewhat different to that elsewhere. Structurally and floristically, the lowland dipterocarp forest of SE Asia is very different to the rainforest of Amazonia, and it is not known what differences this may cause in the speciation and rates of emission of VOCs and hence in atmospheric composition and chemistry. Furthermore, there is strong evidence that transport from the boundary layer in this "maritime continent" region into the upper troposphere, and possibly subsequently into the stratosphere, is particularly efficient (Fueglistaler and Haynes, 2005), so that the region's importance to global atmospheric processes may be disproportionately large.

In common with the other tropical forest regions, SE Asia is undergoing very rapid, and in some cases catastrophic, rates of land use change. For example, in Malaysia, the area of total land cover dedicated to oil palm plantations has increased from $\sim 1 \%$ in 1974 to $\sim 13 \%$ (FAO, 2005; MPOC, 2008). In spite of attempts to implement policies to conserve rainforest, logging of this dwindling resource continues at a rapid rate, and natural forests are being replaced by crop monocultures.

The multi-national OP3 ("Oxidant and particle photochemical processes above a south-east Asian tropical rainforest") project had the goal of better understanding the interactions that exist between natural forests, atmospheric composition and the Earth's climate system. The project had the specific objectives of (a) understanding how emissions of reactive trace gases from a tropical rainforest mediate the regional scale production and processing of oxidants and particles, and (b) better understanding the impacts of these processes on local, regional and global scale atmospheric composition, chemistry and climate. By very closely coupling ground-based and airborne measurements of surface fluxes and atmospheric composition of reactive trace gases and particles with modelling studies of chemical processes, the project aimed to address the following questions:

1. What are the rates of transfer of organic compounds emitted from the tropical forest?

2. How are these organic compounds chemically processed immediately after release?

3. To what extent do the regional organic emissions contribute to the atmospheric aerosol in the region, and what are the effects of the aerosol? What is the composition of the organic fraction of the aerosol?

4. What are the effects of these biogenic emissions on global chemistry and climate? 
The $\mathrm{OH}$ radical initiates the oxidative degradation of biogenically emitted VOCs, and its concentration defines the rate of production of secondary products. A consistent and important finding from field studies conducted in forested environments, characterised by high emissions of isoprene and low levels of $\mathrm{NO}_{\mathrm{x}}$, is the significant underestimation of $\mathrm{OH}$ by models (Lelieveld et al., 2008; Ren et al., 2008; Butler et al., 2008; Carslaw et al., 2001; Martinez et al., 2008; Tan et al., 2001; Kubistin et al., 2009). These model underestimations scale with isoprene concentration and indicate a current inability to correctly describe isoprene oxidation. The OP3 project provided an excellent opportunity to confirm these findings and to seek an explanation. Atmospheric chemistry models, constrained to measured isoprene emission rates, predict dramatic reductions in ambient $\mathrm{OH}$ concentrations in forested areas, in contrast to observations and, as a consequence, predict unrealistically high concentrations of other trace gas constituents (Guenther et al., 2008). Simultaneous measurements of the $\mathrm{OH}$ concentration, isoprene concentration and fluxes and isoprene oxidation products were made during OP3, together with many species that control the rate of production and destruction of $\mathrm{OH}$, providing a stringent set of model constraints to investigate in detail any modelled/measured discrepancies for $\mathrm{OH}$.

Similarly, current models suggest that secondary organic aerosol (SOA) in the tropics is dominated by biogenic aerosol (e.g. Kanakidou et al., 2005), but the measurement database is sparse. Emerging first measurements by aerosol mass spectrometry indicate that sub-micrometre organic aerosol concentrations are at the lower end of the model estimates, with median concentrations of around $1 \mu \mathrm{g} \mathrm{m}^{-3}$ observed in subtropical West Africa and Amazonia (Capes et al., 2009). Despite recent progress, our picture of the formation processes of biogenic SOA (BSOA) is still far from complete (Hallquist et al., 2009). Again, the suite of measurements during OP3 was designed to improve our understanding of the levels, composition and formation processes of BSOA in the SE Asian domain.

As described below, the focus of activity was the Global Atmosphere Watch (GAW) station at Bukit Atur, Sabah, Malaysia, on the island of Borneo $\left(4^{\circ} 58^{\prime} 49.33^{\prime \prime} \mathrm{N}\right.$, $117^{\circ} 50^{\prime} 39.05^{\prime \prime} \mathrm{E}, 426 \mathrm{~m}$ a.s.l.) (http://gaw.empa.ch/gawsis/). Two field campaigns were held during the periods 7 April-4 May 2008 (OP3-I) and 23 June-23 July 2008 (OP3-III). During OP3-III, the UK's largest atmospheric science research aircraft, a converted BAe 146-301, was based at Kota Kinabalu International Airport during the period 8-23 July 2008 and operated for over $60 \mathrm{~h}$ over northern Borneo. Between these two forest campaigns, a sub-set of instruments were deployed in an oil palm plantation, where measurements were made during the period 11 May-20 June 2008 (OP3-II).

In this paper, the land use, vegetation and climate characteristics of the ground-based measurement sites are described, together with an overview of the chemical climatology of the region. The measurement and modelling tools used in the project are also described, as are some preliminary conclusions.

\section{Climate, weather, land use and vegetation of Sabah}

\subsection{Equatorial climate and forest formations}

The equatorial tropics are characterised by rain throughout the year, i.e., an absence of marked seasonal droughts. This climatic regime covers: (i) Malaysia, Papua New Guinea and much of Indonesia within tropical monsoon Asia, (ii) coastal regions of Liberia, Nigeria and Cameroon, and central Congo in Africa, and (iii) western Amazonia and a belt extending from the western Caribbean coast to the Pacific coast in Ecuador in tropical America (McGregor and Nieuwolt, 1998; Walsh, 1996). The equatorial tropics can be further classified into tropical superwet, tropical wet and tropical wet seasonal, using a perhumidity index, based on a cumulative annual score of the number of months with $>200 \mathrm{~mm}(+2$ index value), $100-199 \mathrm{~mm}(+1), 50-99 \mathrm{~mm}(-1)$ and $<50 \mathrm{~mm}$ $(-2)$ of rainfall (Walsh, 1996). The extent of tropical superwet and wet climates (with a perhumidity index $>10$ ) shown in Walsh (1996) is similar to the extent of tropical climates of Asia and America lacking marked dry seasons shown in McGregor and Nieuwolt (1998).

With the exception of the central Congo and the western Caribbean, there is a good correspondence of regions with a tropical wet or superwet climate and the extent of tropical lowland evergreen broadleaf rainforest. This forest formation dominates within the majority of the Asian, West African and American wet/superwet zone of the equatorial tropics (Whitmore, 1998), and is the most common forest formation in the tropics as a whole (Schmitt et al., 2008). However, in areas locally above $750-1200 \mathrm{~m}$ altitude, lowland evergreen broadleaf rainforest grades into lower montane and then upper montane forest. Such areas of mountain forests are noted particularly in the wet/superwet zones of the Asian tropics. Within low-lying areas of the wet and superwet zone, peat swamp, freshwater swamp and heath forest are also present. In areas of podzolic sands, limestone or ultrabasic rocks other forest formations are developed locally.

The key exception to the link between climate and the extent of lowland evergreen rainforest is found within central Congo and the western Caribbean, where tropical semievergreen rainforest dominates in areas classified as tropical wet. This semi-evergreen forest formation is important throughout the surrounding seasonal tropics of continental tropical Asia, north-east Australia, and eastern and southern Amazonia (Whitmore, 1998).

Within the equatorial tropics, the dominant lowland evergreen broadleaf rainforest is characterised by a lofty $(45 \mathrm{~m}$ or taller) and dense canopy with a large number of different tree species occurring together. Usually over two thirds of the upper canopy comprises tree species not contributing 
more than $1 \%$ to the total number (Whitmore, 1998). The soils typically associated with the occurrence of this forest formation in tropical Asia are the Ultisol group, and within western Amazonia the Oxisol group (Baillie, 1996; Chappell et al., 2007).

\subsection{Local climate and forest formations}

The majority of the island of Borneo (total area $743330 \mathrm{~km}^{2}$ ) has a superwet climate (Walsh, 1996) and the most extensive forest formation is lowland evergreen broadleaf rainforest, occupying some $257000 \mathrm{~km}^{2}$ (Schmitt et al., 2008). A similar situation is observed at the $76115 \mathrm{~km}^{2}$ regional scale of the state of Sabah, Malaysian Borneo. Within Borneo Island, and elsewhere within equatorial Asia, the lowland evergreen rainforest typically has a tree family dominance of Dipterocarpaceae (Whitmore, 1984). Most of the state of Sabah was once covered with rainforest (Schmitt et al., 2008), particularly such mixed dipterocarp forest. Currently, some $47 \%$ $\left(36049 \mathrm{~km}^{2}\right)$ of the state lies within Permanent Forest Estate (PFE: Fig. 1a). Most of this PFE (74\%) is maintained under a selective harvesting system (PFE Production Forest), while the remaining 26\% is classified as PFE Protection Forest. Within eastern Sabah, most of the cleared lands are now used for the cultivation of oil palm trees (Fig. 1b). Some commercial timber plantations are also present within Sabah.

The Bukit Atur GAW tower used for OP3 sampling is located within the PFE Production Forest of the Ulu Segama - Malua Forest Reserve, but is less than $5 \mathrm{~km}$ east of the $438 \mathrm{~km}^{2}$ area of PFE Protection Forest known as the Danum Valley Conservation Area (DVCA). The Ulu Segama - Malua Forest Reserve is $2411 \mathrm{~km}^{2}$ in area and is divided into annual timber harvesting coupes. The GAW tower lies at the centre of the $22.6 \mathrm{~km}^{2}$ "Coupe 88 " which was subjected to selective timber harvesting in 1988. An average of $96 \mathrm{~m}^{3}$ of timber ha ${ }^{-1}$ was cut by both tractor and high-lead harvesting (Tangki and Chappell, 2008). The central area of this coupe was subsequently rehabilitated by enrichment planting (Moura-Costa, 1996). As part of a study covering a $225 \mathrm{~km}^{2}$ area Tangki and Chappell (2008) calculated an average tree biomass of $172 \mathrm{tha}^{-1}$ for Coupe 88, (using five inventory plots surveyed in March 1997), and demonstrated a strong correlation $\left(r^{2}=0.76\right)$ between such coupe-averaged values and Landsat-5 TM band 4 (near-infra red) radiance. Tangki (2008) demonstrated that Dipterocarpaceae were the most abundant tree family recorded for the $225 \mathrm{~km}^{2}$ area as a whole (comprising of only lowland evergreen broadleaf rainforest), but Euphobiaceae had become more abundant in Coupe 88 following timber harvesting.

Within the immediate vicinity of the Bukit Atur GAW tower, we calculate the average perhumidity index to be 22 (using the daily rainfall data for the period 1996-2008 from the Danum Valley Field Centre (DVFC), located $8 \mathrm{~km}$ from Bukit Atur at $4^{\circ} 58^{\prime} \mathrm{N}, 117^{\circ} 48^{\prime \prime} \mathrm{E}, 100 \mathrm{~m}$ a.s.l.). Hence Bukit Atur is classified as having a superwet climate. The index
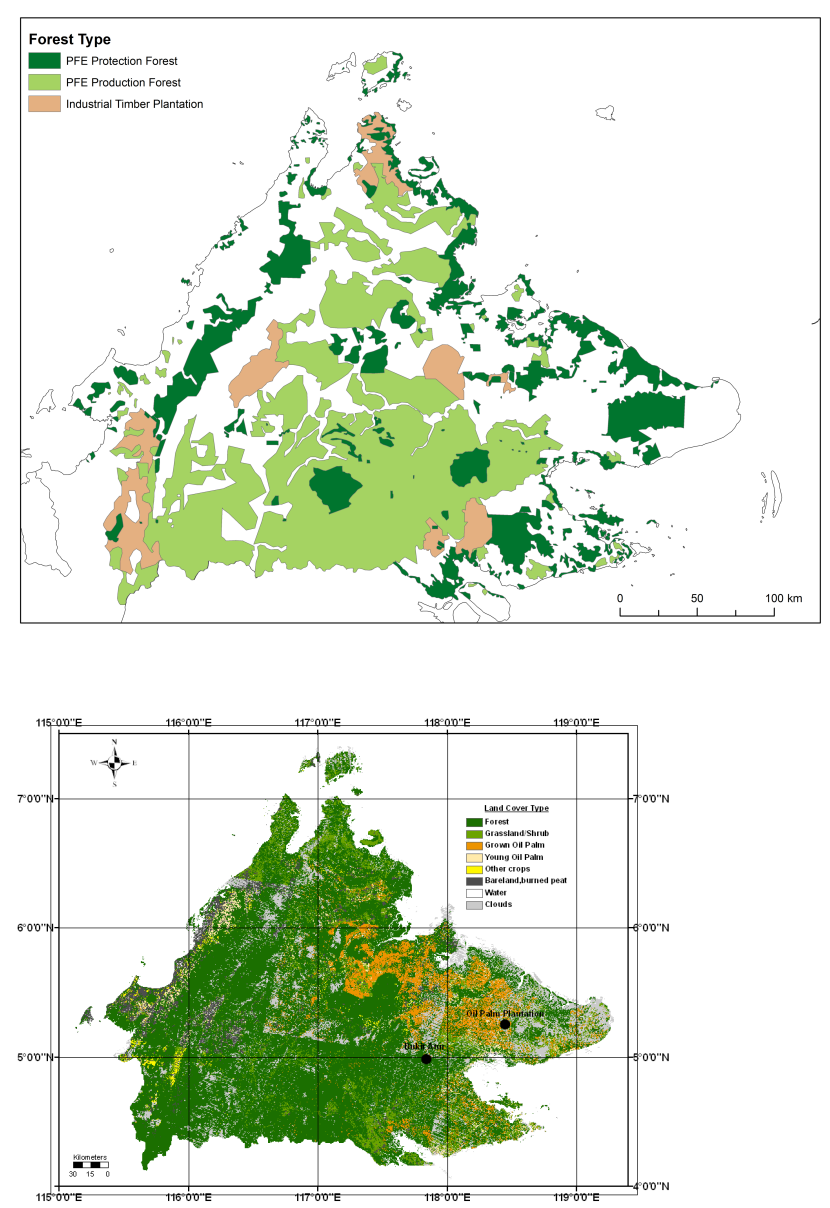

Fig. 1. Land cover maps of Sabah showing (a) the extent of Permanent Forest Estate (PFE) based on Sabah Forest Department (1998) data, where dark green shows PFE Protection Forest, and light green PFE Production Forest; non-PFE commercial timber plantation is also shown in light brown, and (b) extent of oil palm (orange) and other land covers based on a preliminary classification of remote sensing imagery. The satellite data used to produce this map was medium resolution data from Landsat7-ETM+. Eight images from 2005 to 2008 were used. All the images were first geo-referenced using 1:50 000 topographic maps of Sabah. After refinements of the training area collection, the data were reclassified into the eight land cover classes, excluding cloud and shadow.

did however range from 9 (1997) to 23 (1999) as a result of the 4-5 year cycle in the rainfall caused by El Niño South Oscillation phenomena (Chappell et al., 2001). During the OP3 campaign year of 2008 , the perhumidity index was 22 , and therefore identical to the longer-term average.

The 23-year mean rainfall (1986-2008 inclusive) for the DVFC rain gauge is $2840 \mathrm{~mm}( \pm 438 \mathrm{~mm}$ standard deviation). The wettest month is typically January with $310 \mathrm{~mm}$ precipitation. April is typically the driest month with $155 \mathrm{~mm}$ on average, but it also has the most variable rainfall total with a coefficient of variation (CV) of $69 \%$ against 
a mean monthly $\mathrm{CV}$ of $46 \%$. Indeed, the three-month period from February to April has the least predictable rainfall totals, with CV values all over $57 \%$. This variability in rainfall totals may relate to the period being at the change from the northeast monsoon (approximately November-April) to the southwest monsoon (approximately May-October: Bidin and Chappell, 2006).

Rainfall in 2008 totalled $3220 \mathrm{~mm}$, the fifth wettest year in the 23 -year record with $113 \%$ of normal rainfall. The magnitude of the seasonal variations in rainfall was the smallest on record with a $\mathrm{CV}$ of $23 \%$, against the long-term average monthly CV of $47 \%$. This lack of marked seasonality was also shown in the number of days with rainfall. A total of 257 rain-days, the largest number in the 23 -year record, were observed in 2008 against an average of 226 rain-days.

\subsection{Campaign meteorology}

Most of the OP3 measurements were undertaken within the four-month period of April to July 2008. This period was $124 \%$ more wet than normal, with $1045 \mathrm{~mm}$ of rainfall. Notably, the driest month according to the longer-term record, April, received $170 \%$ of the normal rainfall at $263 \mathrm{~mm}$. The April-July 2008 period was also cooler, with a mean temperature of $27.1{ }^{\circ} \mathrm{C}$, which was $99 \%$ of the norm for April-July 2001-2008.

A clear diurnal cycle in the rainfall is observed, even within the records of the relatively short OP3 campaign period of April-July (Fig. 2a). The presence of a late afternoon peak in rainfall at DVFC, which is more pronounced when several years of data are summarised, as in Bidin and Chappell (2006), results from the diurnal development of convective rainfall cells, which is consistent with LIDAR observations (peak rainfall typically observed around 15:00 LT) and measurements of radiation and heat fluxes at the site (Pearson et al., 2010; Helfter et al., 2010). The predominance of rainfall delivery by convective events also results in an extreme localisation of the rainfall field. Within a $5 \mathrm{~km}^{2}$ region encompassing the summit of Bukit Atur, Bidin and Chappell (2003) demonstrated that inter-gauge correlation in annual rainfall totals fell to 0.90 over distances of only $1.1 \mathrm{~km}$, which is short even by comparison with other convective systems.

Figure 2 also shows median temperatures at various heights at Bukit Atur for the combined periods of OP3-I and OP3-III, measured with aspirated thermocouples. The overall median temperature at $30 \mathrm{~m}$ was $25.1 \pm 1.6^{\circ} \mathrm{C}$. This is similar to the long-term surface temperature data from DVFC, taking into account a typical temperature gradient with altitude. During the campaign, the atmospheric stability varied from strongly stable at night to strongly unstable during the middle of the day, as would be expected for a convective region with low wind speeds.

When the boundary layer was stable, the rainforest canopy was decoupled from the overlying atmosphere, resulting
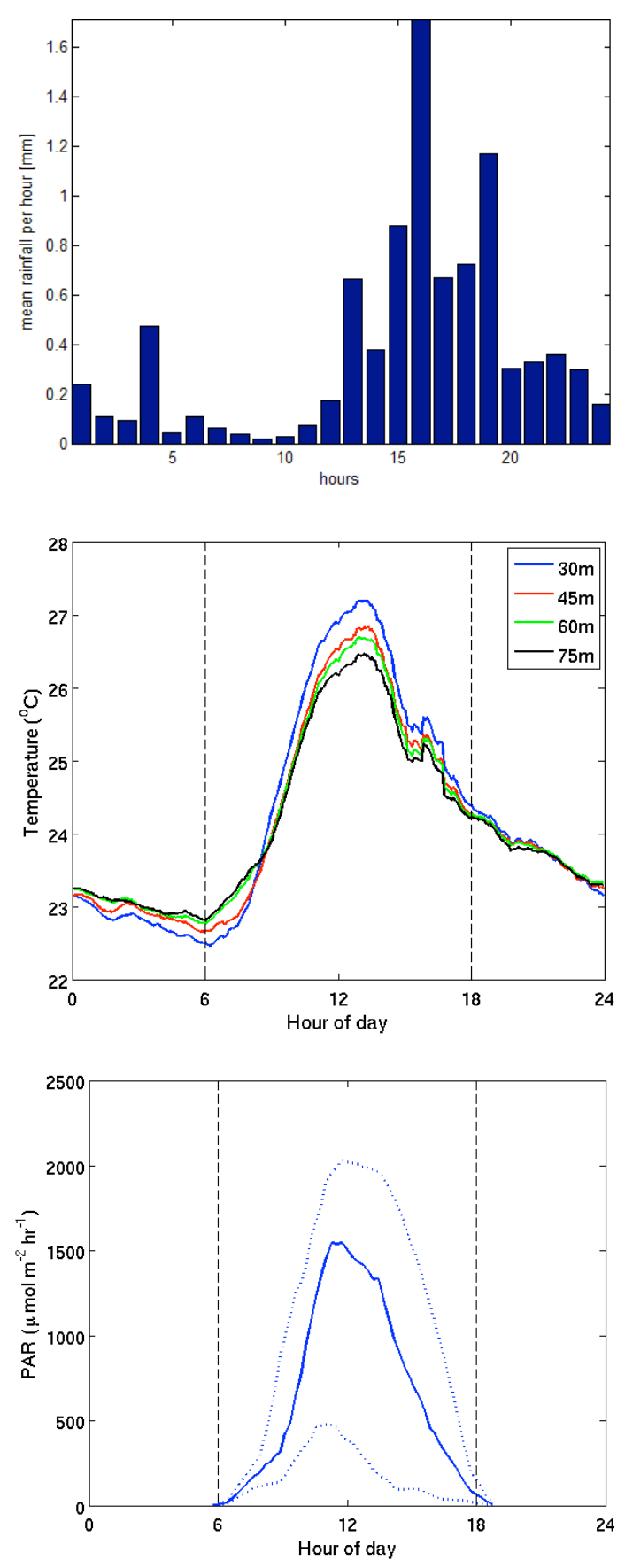

Fig. 2. Local-time diurnal cycles in (top panel) mean rainfall, (middle panel) median temperature at various heights on the Bukit Atur GAW tower, and (bottom) median canopy-top photosynthetically active radiation (PAR), over the OP3 campaign months of AprilJuly 2008. Dashed lines on temperature and PAR graphs show approximate sunrise and sunset times. Dotted lines on the PAR graph show 5 th and 95 th percentiles.

very frequently in nocturnal radiation fogs. LIDAR data (not shown) demonstrate this, and also suggest a day-time (10:00-18:00 LT) mixing height of $\sim 800 \mathrm{~m}$. The strong 
daytime turbulence, along with weak winds, makes chemical box-modelling a reasonable strategy for interpretation of daytime atmospheric composition (Sect. 5, below), but understanding measurements made at night, when the atmosphere near the surface is strongly stratified, requires more careful consideration of vertical mixing (Pugh et al., 2010a).

The bottom panel of Fig. 2 shows the diurnal cycle in photosynthetically active radiation (PAR), measured at canopytop height. There is a tail of dull days (shown by the 5th percentile) but, on the whole, the period of the campaign was bright. The warm temperatures and bright sunshine produced substantial emissions of biogenic volatile organic compounds (BVOCs) from the forest (Sect. 4.2.2, below).

Turning from vertical mixing/convective effects, to take a more horizontal/advective view, backwards air mass trajectories were analysed in order to characterise the origins of chemical species measured at Bukit Atur during the measurement campaigns. They were calculated by the British Atmospheric Data Centre (BADC) Web Trajectory Service using European Centre for Medium-Range Weather Forecasts (ECMWF) wind fields. A series of trajectories was calculated, with one trajectory for every hour during OP3-I and OP3-III. These were then analysed to give an ensemble representation of air mass residency time as a function of location for the whole of each campaign (Ashbaugh et al., 1985). Back trajectories are calculated for the $24 \mathrm{~h}$ before arrival at Bukit Atur, with a time resolution of $30 \mathrm{~min}$, and a final pressure altitude of $950 \mathrm{hPa}$. Back trajectories that touch the ground have been removed. Figure $3 \mathrm{a}$ and $\mathrm{b}$ show air mass residency time on a $0.1^{\circ} \times 0.1^{\circ}$ grid for all back trajectories from the first and third campaign periods, respectively.

The first campaign period (OP3-I) was influenced by air masses from most directions, in contrast to OP3-III when the air was predominantly from the south. The southern air in the third period can be split into two main areas of origin: the SE air from the sea with a minimal fetch over land, and the SW air which is exclusively over land. This can be used to identify and compare periods of marine and terrain influenced air. It is possible to extend this analysis by using only trajectories from periods when a certain measurement is elevated, giving a coloured probability distribution of the source of the measured quantity. A subsequent paper will use these techniques to present a more in-depth analysis of chemical origins in the future.

\subsection{Land-cover classification and VOC emissions modelling}

Biogenic VOC emissions are highly sensitive to land-cover characteristics and can vary over several orders of magnitude across different landscapes. This is partly due to variability in total biomass density but is greatly enhanced by variability among different plant species, especially for compounds such as isoprene that are emitted by less than a third of all plant species. This presents a daunting challenge for at-
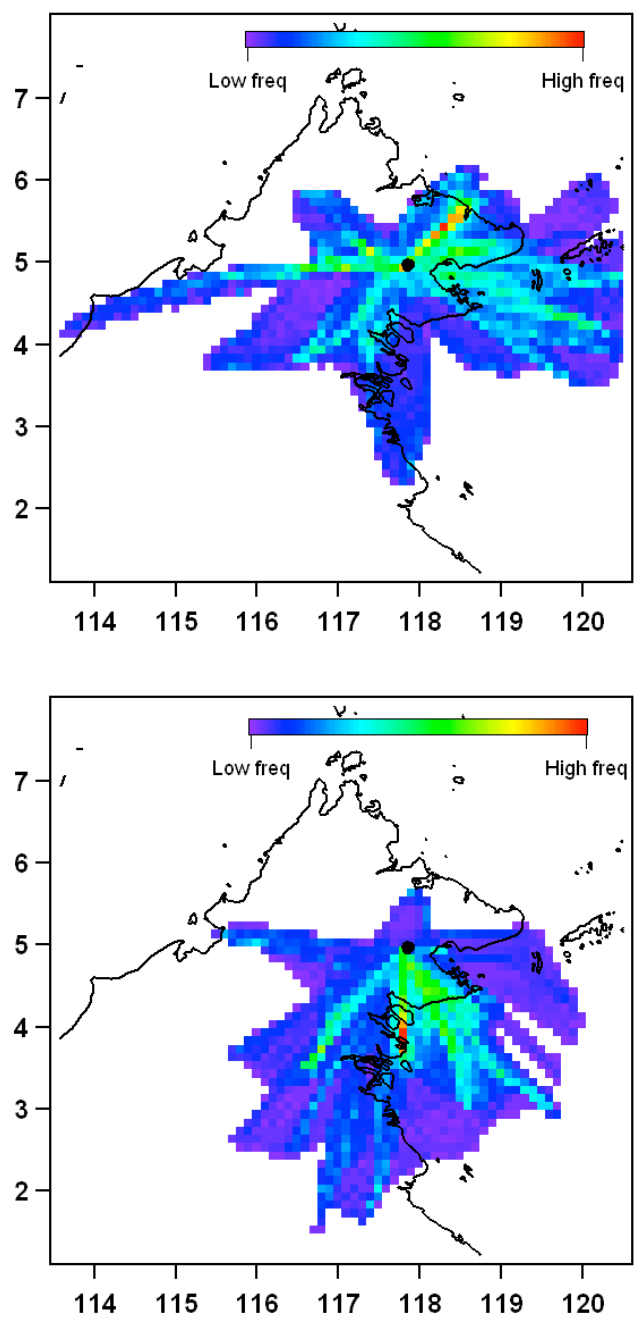

Fig. 3. Air mass residency times for air reaching Bukit Atur (black circle) during (top panel) OP3-I and (bottom panel) OP3-III. No colour means no trajectories passed over that area in the last $24 \mathrm{~h}$.

tempts to characterize regional BVOC emissions, especially in highly diverse tropical forests.

Guenther et al. (1995) estimated global biogenic VOC emissions using the highest resolution $\left(0.5^{\circ}\right)$ and most detailed global map of land-cover and land-use (Olson, 1992) available at that time. Figure 4 (top left panel) illustrates the Olson (1992) classification of Borneo landscapes, which include six unmanaged land-cover types, dominated by tropical rainforest (50\%), marsh/swamp (14\%) and tropical montane $(7 \%)$ and three managed land-cover types dominated by re-growing woods $(9 \%)$ and paddy rice $(7 \%)$. The associated isoprene emission factor map shown in Fig. 4 (top right panel) characterizes the gross features of Borneo but does not represent the full diversity of landscapes in this region. A major limitation of the Olson global ecosystem approach is that, for example, all re-growing woods are lumped together and observations from North American and European forests 

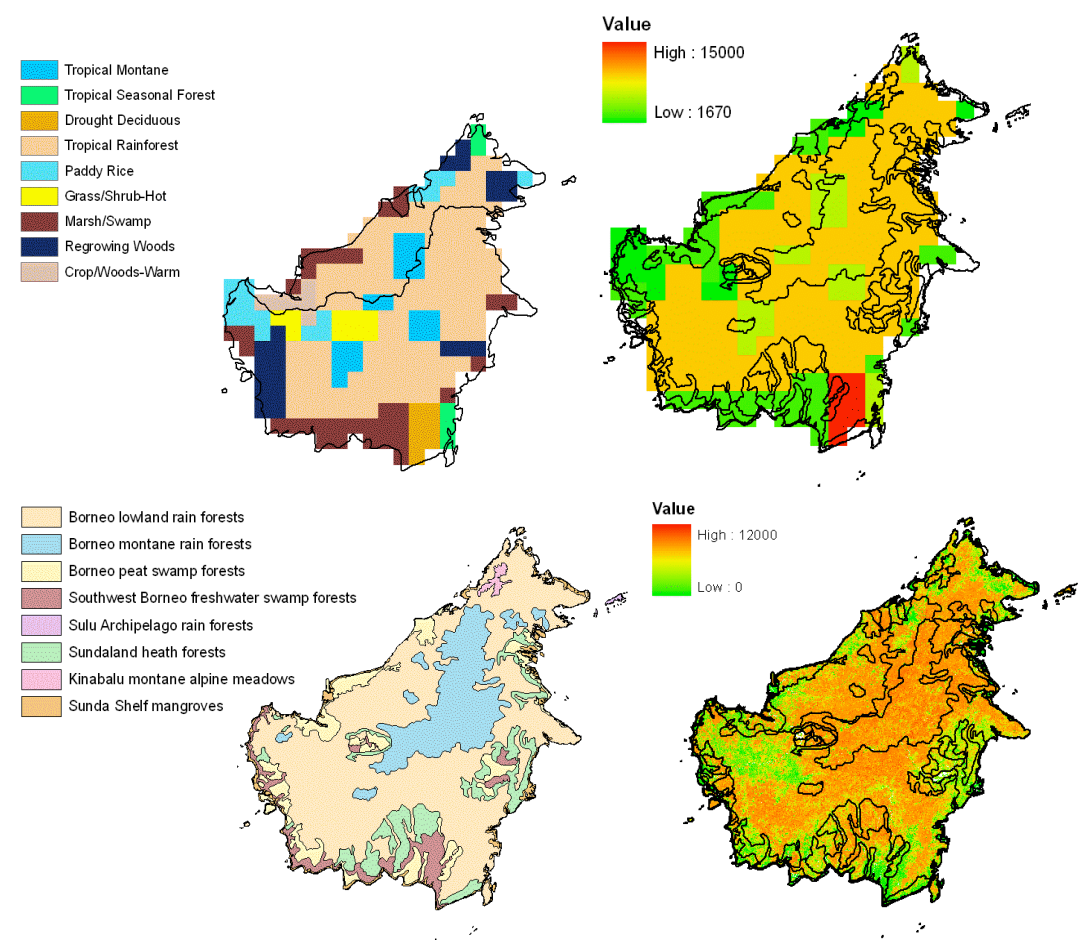

Fig. 4. Land cover distributions for Borneo used by the global biogenic VOC emissions inventory of Guenther et al., 1995) (top left) and by MEGAN (Guenther et al., 2006) (bottom left) and associated isoprene emission factor $\left(\mu \mathrm{g} \mathrm{m}^{-2} \mathrm{~h}^{-1}\right.$ ) map used by Guenther et al. (1995) (top right) and by MEGAN (Guenther et al., 2006) (bottom right).

were used to assign a single isoprene emission factor to all occurrences of this land-cover type across the globe. The Guenther et al. (1995) estimate of annual isoprene emission from Borneo is about $10 \mathrm{Tg}$ of carbon, which is $2 \%$ of the estimated global total.

The availability of satellite observations has greatly improved quantitative estimates of eco-region distributions and other land-cover variables, including leaf area indices (LAI) and plant functional type (PFT) cover fractions. In addition, the development of a global geo-referenced eco-region map by Olson et al. (2001) represents an additional major advance for biogenic emission modeling. This high resolution digital map is the product of over 1000 biogeographers, taxonomists, conservation biologists and ecologists from around the world. Each of the 867 eco-regions represented on this map has relatively uniform species composition and is accompanied by an online database (http: //www.nationalgeographic.com/wildworld/) that includes a description of the dominant plant species. Figure 4 (lower left) shows that this database divides Borneo into seven ecoregions with Borneo lowland rainforests covering just over half of the total land area, Borneo montane rainforests and Sundaland heath forests together comprise about $25 \%$, and the remaining four ecoregions (Kinabalu montane alpine meadows, Borneo peat swamp forests, Borneo freshwater swamp forests, Sunda Shelf mangroves) each make up 1 to
$8 \%$ of the total. While the total area associated with broad types (e.g. tropical forest, montane forest) agree reasonably well with the Olson (1992) database, the details differ considerably. Figure 4 (bottom right) shows that land-cover results in considerable differences between the isoprene emission factor distribution of Guenther et al. (1995) and the MEGAN model (Guenther et al., 2006) which uses the Olson et al. (2001) eco-region map and satellite derived PFT (e.g. crop, broadleaf tree, shrub) cover fractions. In addition, MEGAN uses plant species composition estimates from the Olson et al. (2001) global terrestrial eco-region database and the Leff et al. (2004) crop species distribution database. Although the spatial pattern is quite different, the annual isoprene emission for Borneo differs by less than $5 \%$ when the MEGAN land-cover is used in place of Guenther et al. (1995) land-cover data. This good agreement, however, is the result of major offsetting differences between these two land-cover databases. The Guenther et al. (1995) foliar density and LAI estimates are about $50 \%$ higher, resulting in about $25 \%$ more isoprene, but the emission factors are about $25 \%$ lower. The result is a very similar annual isoprene emission but for different reasons.

The MEGAN framework can be used to estimate regional to global biogenic VOC emissions but the accuracy of the results is dependent on the availability of representative measurements of individual ecoregions. A lack of BVOC 
measurements from Borneo resulted in the assignment of MEGAN version 2.1 emission factors to the ecoregions of Borneo that were based on observations from other tropical regions. Improved estimates for future versions of MEGAN and other models are highly dependent on the availability of observations characterizing the dominant plant functional types within major global ecoregions. In fact, our initial analysis of emissions from Bukit Atur show that the default base emission rates in MEGAN prior to the OP3 observations are a factor of four too high for this forest ecoregion. We also confirm that, unusually for a species classified as crop in MEGAN, oil palm is an intense isoprene emitter.

\section{Measurement strategy and methods}

The overall measurement strategy for OP3 was to perform integrated measurements from the forest-floor, through the forest canopy, above the canopy and then up-scaled to the regional scale using airborne measurements, with clear linkages between measurements made at the different sites at the different scales.

\subsection{Ground based measurements}

Ground based measurements were centred on the $100 \mathrm{~m}$ tower at the Bukit Atur GAW station $\left(4^{\circ} 58^{\prime} 49.33^{\prime \prime} \mathrm{N}\right.$, $117^{\circ} 50^{\prime} 39.05^{\prime \prime} \mathrm{E}, 426 \mathrm{~m}$ a.s.l.) (http://gaw.empa.ch/gawsis/). An "in-canopy" sampling site was established $2 \mathrm{kmE}$ of the Bukit Atur tower $\left(4^{\circ} 58^{\prime} 49.10^{\prime \prime} \mathrm{N}, 117^{\circ} 51^{\prime} 19.12^{\prime \prime} \mathrm{E}\right)$ and a further "oil palm plantation" sampling site was established at the Sabahmas oil palm estate, $70 \mathrm{~km} \mathrm{NE}$ of Bukit Atur $\left(5^{\circ} 14^{\prime} 58.69^{\prime \prime} \mathrm{N}, 118^{\circ} 27^{\prime} 15.76^{\prime \prime} \mathrm{E}\right)$.

\subsubsection{Forest floor (soil) $\mathrm{NO}_{\mathrm{x}}$ flux measurements}

The fluxes of $\mathrm{NO}_{\mathrm{x}}$ from forest soil were measured using a continuous automated dynamic chamber system at the "incanopy site". Seven spatially distributed chambers were used in order to represent the spatial variation inherent in soil $\mathrm{NO}_{\mathrm{x}}$ emissions at each site. The chamber construction and operation is described in Pilegaard (2001) while the flux calculation method was modified from Conrad (1994), with further details available in Dorsey and Gallagher (2010). In addition, fluxes of nitrous oxide $\left(\mathrm{N}_{2} \mathrm{O}\right)$ and methane $\left(\mathrm{CH}_{4}\right)$ were made at the "in-canopy site", at a near-by undisturbed primary forest site, at a heavily disturbed road-side site and from the soils at the oil palm plantation (see below), using static soil chambers (Siong et al., 2009; Ryder et al., 2010).

\subsubsection{Within-canopy concentration profiles}

At the "in-canopy site", measurements were made of the vertical gradients (from the ground to $32 \mathrm{~m}$ height) of the concentrations of ozone, $\mathrm{NO}_{\mathrm{x}}$, volatile organic compounds, particle size distribution and composition, as well as temperature, relative humidity, radiation and turbulence. Four platforms were strapped against an emergent tree (Canarium decumanum), at $8,16,24,32 \mathrm{~m}$. Each platform supported sonic anemometers, inlets to a gradient system for $\mathrm{O}_{3} / \mathrm{NO} / \mathrm{NO}_{2}$ and fine thermocouples. In addition, an automated winch system continuously raised and lowered a temperature/humidity probe, a PAR sensor, an optical particle spectrometer (GRIMM 1.08) and an inlet leading to a PTRMS. This gave vertical gradient measurements between 2 and $28 \mathrm{~m}$ (Ryder et al., 2010). In a clearing near the in-canopy site, LIDAR measurements were made of wind speed and direction and of aerosol backscatter throughout the boundary layer.

\subsubsection{Concentration and flux measurements at Bukit Atur}

The largest number of ground-based measurements were made at the Bukit Atur GAW station, which routinely records $\mathrm{CO}_{2}$ and $\mathrm{O}_{3}$ mixing ratios, and various aerosol parameters. The station consists of a main building with four airconditioned laboratories at the base of a $100 \mathrm{~m}$ tower, all located in a large $(\sim 150 \times 50 \mathrm{~m})$ clearing on the top of a hill and surrounded by forest. The surrounding forest canopy extends $\sim 10 \mathrm{~m}$ above the base of the tower. Four mobile seacontainer laboratories were deployed around the base of the tower to provide extra instrument accommodation. Electrical power was provided by generators, located $2 \mathrm{kmE}$ of the station. Pollution events, attributable to individual vehicles arriving on site, the generators, or to small leaks of reactive compounds on site, were identified by elevated concentrations of the oxides of nitrogen and by wind direction analysis, and are excluded from subsequent data analysis. Table 1 summarises the measurements made at Bukit Atur: the critical measurements included:

- eddy covariance and gradient flux measurements of trace gases and particles;

- speciated concentration measurements of trace gases and particles;

- measurements of aerosol size-dependent hygroscopicity and critical supersaturations for cloud growth;

- concentration measurements of $\mathrm{OH}, \mathrm{HO}_{2}$, and the sum of hydroperoxy and organic peroxy radicals;

- $\mathrm{OH}$ reactivity measurements (the rate at which $\mathrm{OH}$ is removed from the atmosphere);

- characteristics of boundary layer turbulence and mixing. 


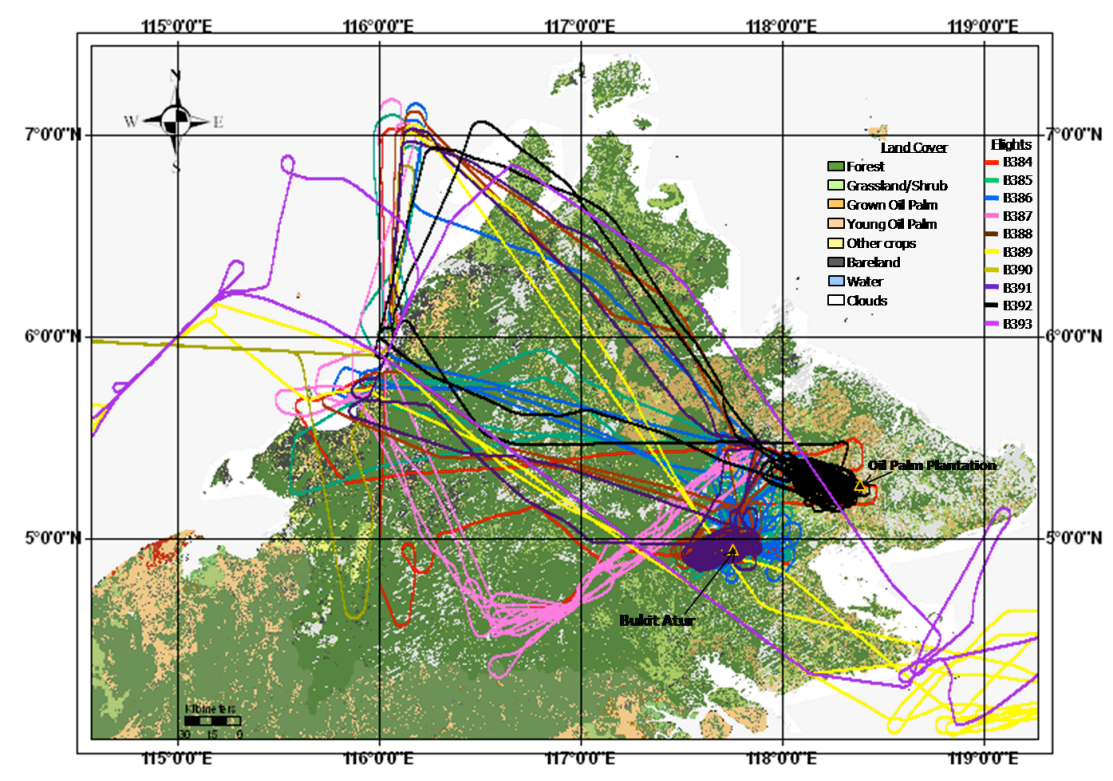

Fig. 5. Flight tracks of the BAe 146 research aircraft over Sabah during OP3-III. The underlying map is as shown in Fig. 1b.

\subsubsection{Concentration and flux measurements at the Sabamas oil palm plantation}

The Sabahmas oil palm plantation measurement site used during OP3-II was located in a 33 ha flat area of oil palm (Elaeis guineensis $\times$ Elaeis oleifera hybrid, progeny "Guthrie") trees. The trees were of uniform age (12 years) and height $(12 \mathrm{~m})$. The site comprised a $15 \mathrm{~m}$ tower and an $8 \mathrm{~m}$ canopy access platform. Instruments were housed in a hut at the base of the platform. The analytical methods used were the same as at Bukit Atur, including the measurements of aerosol sub-micrometre composition, fluxes of aerosol, BVOCs, ozone and $\mathrm{CO}_{2}$, although the suite of measurements made was not as comprehensive. In particular, measurements of $\mathrm{OH}$ and other radicals and of the oxides of nitrogen were not made.

\subsubsection{Airborne measurements}

Airborne measurements (see Table 1) were made during OP3-III using the Natural Environment Research Council/UK Meteorological Office's BAe 146-301 Facility for Airborne Atmospheric Measurements (FAAM) aircraft (Lewis et al., 2007), deployed to Kota Kinabalu airport, less than 30 min flying time from Bukit Atur. In general, the same type of flight plan was executed on each flight, with one profile up and one down, interrupted by straight and level runs at altitudes of 100-250, 1500, 3000 and $6000 \mathrm{~m}$ above ground over the rainforest (centred on Bukit Atur), over an extensive and homogeneous agro-industrial oil palm landscape surrounding and including the Sabahmas oil palm plantation estate, and in transects between the two sites. Flights were made morning and afternoon giving typically four stacked profiles, allowing a picture to be built up of the concentrations of trace gases and particles during the daytime. Two flights were also made over the ocean up- and down-wind of Sabah.

In order to link the ground-based and airborne measurements, the aircraft flew past the Bukit Atur measurement station on more than ten occasions, at the same height above sea-level as the base of the GAW tower and at approximately $500 \mathrm{~m}$ horizontal separation. Table 2 summarises the flights made during the OP3 deployment and Fig. 5 shows the geographical extent of the flights over Sabah.

\section{The chemical climatology of Sabah and Bukit Atur}

\subsection{Satellite observations}

Satellite observations of key tropospheric trace gases allow the surface and aircraft trace gas measurements above the rainforest surrounding Bukit Atur to be put in a wider context relative to Borneo and the larger south-east Asian region. We focus on formaldehyde (HCHO) and nitrogen dioxide $\left(\mathrm{NO}_{2}\right)$, which are good indicators of emissions and photochemical activity, to examine the atmospheric chemistry over Borneo during the OP3 campaigns in 2008. Global background concentrations of $\mathrm{HCHO}$ are determined by the balance between the source (from the oxidation of methane) and the $\mathrm{OH}$ sink. Concentrations are typically much larger over continents due to additional sources from the oxidation of biogenic and anthropogenic VOCs, and from biomass burning (either directly released or from the oxidation of co-emitted VOCs) (Palmer et al., 2003; Fu et al., 2007). Anthropogenic activities, biomass burning and soil emissions are the main source 
Table 1. Overview of the measurements made in OP3.

\begin{tabular}{|c|c|c|c|c|c|c|c|}
\hline Species & $\begin{array}{l}\text { Method/ } \\
\text { Analytical } \\
\text { Technique }\end{array}$ & Ground $\dagger$ & Air & $\begin{array}{l}\text { Temporal } \\
\text { Resolution }\end{array}$ & $\begin{array}{l}\text { Detection } \\
\text { Limit }\end{array}$ & $\begin{array}{l}\text { Measurement } \\
\text { Uncertainty }\end{array}$ & Reference \\
\hline $\begin{array}{l}\text { NMHC, includ- } \\
\text { ing isoprene } \\
\text { and oxygenates }\end{array}$ & $\begin{array}{l}\text { Disjunct eddy } \\
\text { covariance flux } \\
\text { measurement } \\
\text { with continu- } \\
\text { ous flow and } \\
\text { analysis by } \\
\text { PTR-MS }\end{array}$ & $\sqrt{ } \mathrm{BA}, \mathrm{OP}$ & & $\begin{array}{l}\text { Fluxes: } \\
30 \text { min } \\
\text { Mixing } \\
\text { ratios: } \sim 7 \mathrm{~s}\end{array}$ & $\begin{array}{l}\text { Fluxes: } \\
<0.05 \\
\text { mg m }^{-2} \mathrm{~h}^{-1} \\
\text { Mixing ra- } \\
\text { tios: } 10-100 \\
\text { pptv }\end{array}$ & $\begin{array}{l}\text { Fluxes: } \\
\text { Precision }= \\
\sim \pm 30 \% \\
\text { Mixing ratios } \\
\sim \pm 10 \%\end{array}$ & $\begin{array}{l}\text { (Langford et } \\
\text { al., 2009) }\end{array}$ \\
\hline $\begin{array}{l}\text { NMHC, includ- } \\
\text { ing isoprene } \\
\text { and oxygenates }\end{array}$ & $\begin{array}{l}\text { Automated } \\
\text { PTR-MS } \\
\text { gradient }\end{array}$ & $\sqrt{ } \mathrm{IC}$ & & $\begin{array}{l}\text { Gradient ev- } \\
\text { ery } 7 \mathrm{~min}\end{array}$ & $10-100$ pptv & & $\begin{array}{l}\text { (Karl et al., } \\
\text { 2007) }\end{array}$ \\
\hline $\begin{array}{l}\text { NMHC, includ- } \\
\text { ing isoprene, } \\
\text { monoterpenes } \\
\text { and oxygenates }\end{array}$ & $\begin{array}{l}\text { Dual Channel } \\
\text { Gas Chro- } \\
\text { matograph } \\
\text { with } \\
\text { Flame ionisa- } \\
\text { tion detectors } \\
\text { (DC-GC-FID) }\end{array}$ & $\sqrt{ } \mathrm{BA}$ & $\sqrt{ }$ & $\begin{array}{l}1 \mathrm{~h} \text { ground } \\
\text { variable air }\end{array}$ & $1 \mathrm{pptv}$ & $\begin{array}{l}\text { variable, typ- } \\
\text { ically around } \\
10 \%\end{array}$ & $\begin{array}{l}\text { (Lewis et al., } \\
\text { 2007; Lewis et } \\
\text { al., 2005) }\end{array}$ \\
\hline $\begin{array}{l}\text { Terpenoids, al- } \\
\text { cohols, aldehy- } \\
\text { des }\end{array}$ & $\begin{array}{l}\text { GC-PID, } \\
\text { portable mass } \\
\text { spectrometer }\end{array}$ & $\sqrt{ } \mathrm{BA}$ & & & & & \\
\hline $\mathrm{CO}_{2} / \mathrm{H}_{2} \mathrm{O}$ flux & $\begin{array}{l}\text { Eddy- } \\
\text { covariance } \\
\text { flux using } \\
\text { infrared gas } \\
\text { analyser Li-Cor } \\
7000 / 7500\end{array}$ & $\sqrt{ } \mathrm{BA}, \mathrm{OP}$ & & 30 min fluxes & & & $\begin{array}{l}\text { (Aubinet et al., } \\
2000 \text { ) }\end{array}$ \\
\hline $\mathrm{NO}_{2}$ flux & $\begin{array}{l}\text { Eddy covari- } \\
\text { ance using laser } \\
\text { induced } \\
\text { fluorescence }\end{array}$ & $\sqrt{ } \mathrm{BA}$ & & 30 min fluxes & & & $\begin{array}{l}\text { (Farmer et al., } \\
\text { 2006) }\end{array}$ \\
\hline $\begin{array}{l}\mathrm{HNO}_{3}, \mathrm{HCl}, \\
\mathrm{HNO}_{2}, \mathrm{NH}_{3}, \\
\mathrm{SO}_{2}, \mathrm{NH}_{4}^{+}, \\
\mathrm{NO}_{3}^{-}, \mathrm{Cl}^{-}, \\
\mathrm{SO}_{4}^{2-}\end{array}$ & $\begin{array}{l}\text { Wet effluent } \\
\text { denuder \& } \\
\text { steam jet } \\
\text { aerosol collec- } \\
\text { tor, online IC } \\
\text { (GRAEGOR) }\end{array}$ & $\sqrt{ } \mathrm{BA}$ & & $1 \mathrm{~h}$ & & & $\begin{array}{l}\text { (Thomas et al., } \\
\text { 2009) }\end{array}$ \\
\hline $\begin{array}{l}\text { Turbulence, } \\
\text { sensible heat } \\
\text { flux }\end{array}$ & $\begin{array}{l}\text { Eddy- } \\
\text { covariance } \\
\text { using sonic } \\
\text { anemometry }\end{array}$ & $\sqrt{ } \mathrm{BA}$ & & $30 \mathrm{~min}$ fluxes & & & \\
\hline Soil NO flux & $\begin{array}{l}\text { Dynamic auto- } \\
\text { chamber using } \\
\text { NO analyser }\end{array}$ & $\sqrt{ } \mathrm{IC}$ & & & & & $\begin{array}{l}\text { (Pilegaard et } \\
\text { al., 2006) }\end{array}$ \\
\hline
\end{tabular}


Table 1. Continued.

\begin{tabular}{|c|c|c|c|c|c|c|c|}
\hline Species & $\begin{array}{l}\text { Method/ } \\
\text { Analytical } \\
\text { Technique }\end{array}$ & Ground $\dagger$ & Air & $\begin{array}{l}\text { Temporal } \\
\text { Resolution }\end{array}$ & $\begin{array}{l}\text { Detection } \\
\text { Limit }\end{array}$ & $\begin{array}{l}\text { Measurement } \\
\text { Uncertainty }\end{array}$ & Reference \\
\hline $\begin{array}{l}\text { Soil } \mathrm{N}_{2} \mathrm{O} / \mathrm{CH}_{4} \\
\text { flux }\end{array}$ & $\begin{array}{l}\text { Static soil } \\
\text { chamber with } \\
\text { off-line GC } \\
\text { analysis }\end{array}$ & $\sqrt{ } \mathrm{IC}, \mathrm{OP}$ & & $1 \mathrm{~h}$ & & & \\
\hline $\mathrm{OH}, \mathrm{HO}_{2}$ & $\begin{array}{l}\text { FAGE } \\
\text { (Fluorescence } \\
\text { Assay by Gas } \\
\text { Expansion) } \\
\text { laser-induced } \\
\text { fluorescence }\end{array}$ & $\sqrt{ } \mathrm{BA}$ & & $10 \mathrm{~s}$ & $\begin{array}{l}(\mathrm{OH}) \\
2.4 \times 10^{5} \\
\text { molecule } \\
\mathrm{cm}^{-3}(3 \mathrm{~min} \\
\text { av. }) \\
\left(\mathrm{HO}_{2}\right) \\
3.8 \times 10^{6} \\
\text { molecule }^{-3}(3 \mathrm{~min} \\
\mathrm{cm}^{-3} \\
\text { av. })\end{array}$ & $\begin{array}{l}44 \%(\mathrm{OH}) \\
50 \%\left(\mathrm{HO}_{2}\right) \\
(2 \sigma)\end{array}$ & $\begin{array}{l}\text { (Whalley et al., } \\
\text { 2010a, b) }\end{array}$ \\
\hline $\mathrm{OH}, \mathrm{HO}_{2}$ & $\begin{array}{l}\text { FAGE } \\
\text { (Fluorescence } \\
\text { Assay by Gas } \\
\text { Expansion) } \\
\text { laser-induced } \\
\text { fluorescence }\end{array}$ & & $\sqrt{ }$ & $60 \mathrm{~s}$ & $\begin{array}{l}(\mathrm{OH}) \\
2.3 \times 10^{6} \\
\text { molecule } \\
\mathrm{cm}^{-3}(1 \mathrm{~min} \\
\text { av. }) \\
\left(\mathrm{HO}_{2}\right) \\
2.0 \times 10^{6} \\
\mathrm{molecule}^{6} \\
\mathrm{~cm}^{-3}(1 \mathrm{~min} \\
\text { av. })\end{array}$ & $\begin{array}{l}28 \%(\mathrm{OH} \\
\left.\& \mathrm{HO}_{2}\right)(2 \sigma)\end{array}$ & \\
\hline OH Reactivity & FAGE & $\sqrt{ } \mathrm{BA}$ & & $1 \mathrm{~min}$ & & $22 \%(2 \sigma)$ & $\begin{array}{l}\text { (Ingham et al., } \\
\text { 2009) }\end{array}$ \\
\hline $\begin{array}{l}\Sigma \mathrm{RO}_{2}+\mathrm{HO}_{2} \\
\mathrm{HO}_{2}\end{array}$ & $\begin{array}{l}\text { PERCA (PEr- } \\
\text { oxy Radical } \\
\text { Chemical Am- } \\
\text { plifier), dual in- } \\
\text { let }\end{array}$ & $\sqrt{ } \mathrm{BA}$ & $\sqrt{ }$ & $1 \mathrm{~min}$ & $\begin{array}{l}0.4 \mathrm{pptv} \\
(10 \mathrm{~min})\end{array}$ & $38 \%(1 \sigma)$ & $\begin{array}{l}\text { (Fleming et al., } \\
\text { 2006) }\end{array}$ \\
\hline $\begin{array}{l}\mathrm{NO}_{3}, \mathrm{CH}_{2} \mathrm{O}, \\
\mathrm{NO}_{2}, \mathrm{HONO}, \\
\mathrm{O}_{3}, \mathrm{CHOCHO}\end{array}$ & $\begin{array}{l}\text { Differential } \\
\text { Optical Ab- } \\
\text { sorption } \\
\text { Spectroscopy }\end{array}$ & $\sqrt{ } \mathrm{BA}$ & & $10 \mathrm{~min}$ & $\begin{array}{l}2 \text { pptv, } 480 \\
\text { pptv, } 80 \text { pptv, } \\
150 \text { pptv, } 4.6 \\
\text { ppbv, } \\
150 \text { pptv }\end{array}$ & $\begin{array}{l}1.5 \text { pptv, } \\
500 \text { pptv, } 60 \\
\text { pptv, } 130 \text { pptv, } \\
4 \text { ppbv, } 130 \\
\text { pptv }\end{array}$ & $\begin{array}{l}\text { (Plane and } \\
\text { Saiz-Lopez, } \\
\text { 2006) }\end{array}$ \\
\hline $\begin{array}{l}\mathrm{NO}_{2}, \mathrm{HCHO}, \\
\text { CHOCHO }\end{array}$ & MAX-DOAS & $\sqrt{ } \mathrm{BA}$ & & & & & $\begin{array}{l}\text { (Leigh et al., } \\
\text { 2006) }\end{array}$ \\
\hline $\begin{array}{l}\text { Photolysis fre- } \\
\text { quencies (incl. } \\
\left.\mathrm{j}\left(\mathrm{O}^{1} \mathrm{D}\right), \mathrm{j}\left(\mathrm{NO}_{2}\right)\right)\end{array}$ & $\begin{array}{l}\text { Calibrated filter } \\
(2 \pi \text { and } 4 \pi \\
\text { sr) radiometers } \\
\text { and spectral- } \\
\text { radiometer }\end{array}$ & $\sqrt{ } \mathrm{BA} / \mathrm{IC}$ & $\sqrt{ }$ & $1 \mathrm{~s}$ & $\mathrm{n} / \mathrm{a}$ & $\begin{array}{l}14 \% \text { and } 13 \% \\
0-90^{\circ} \text { SZA }\end{array}$ & $\begin{array}{l}\text { (Bohn et al., } \\
\text { 2008; Edwards } \\
\text { and Monks, } \\
\text { 2003; Volz- } \\
\text { Thomas, et al., } \\
\text { 1996) }\end{array}$ \\
\hline $\mathrm{O}_{3}$ & UV absorption & $\sqrt{ } \mathrm{BA}$ & $\sqrt{ }$ & $1 \mathrm{~s}$ & $0.6 \mathrm{ppbv}$ & $\begin{array}{l}10 \% \pm 3.4 \mathrm{ppbv} \\
( \pm 1 \sigma)\end{array}$ & $\begin{array}{l}\text { (Heard et al., } \\
\text { 2006) }\end{array}$ \\
\hline
\end{tabular}


Table 1. Continued.

\begin{tabular}{|c|c|c|c|c|c|c|c|}
\hline Species & $\begin{array}{l}\text { Method/ } \\
\text { Analytical } \\
\text { Technique }\end{array}$ & Ground $\dagger$ & Air & $\begin{array}{l}\text { Temporal } \\
\text { Resolution }\end{array}$ & $\begin{array}{l}\text { Detection } \\
\text { Limit }\end{array}$ & $\begin{array}{l}\text { Measurement } \\
\text { Uncertainty }\end{array}$ & Reference \\
\hline $\begin{array}{l}\mathrm{O}_{3} \text { flux eddy } \\
\text { correlation }\end{array}$ & $\begin{array}{l}\text { Dry chemilu- } \\
\text { minescence }\end{array}$ & $\sqrt{ } \mathrm{BA}, \mathrm{OP}$ & & $\begin{array}{l}30 \text { min fluxes } \\
\text { from } 0.05 \mathrm{~s}\end{array}$ & $0.1 \mathrm{ppbv}$ & & $\begin{array}{l}\text { (Güsten et al., } \\
\text { 1990; Gusten } \\
\text { and Heinrich, } \\
\text { 1996) }\end{array}$ \\
\hline $\begin{array}{l}\mathrm{O}_{3} / \mathrm{NO} / \mathrm{NO}_{2} \\
\text { gradient }\end{array}$ & $\begin{array}{l}\text { Chemilu- } \\
\text { minescence } \\
\left(\mathrm{O}_{3}\right) \text {, thermal } \\
\text { converter }\end{array}$ & $\sqrt{ } \mathrm{BA} / \mathrm{IC}$ & & $15 \mathrm{~min}$ & & & \\
\hline $\begin{array}{l}\mathrm{NO} \\
\mathrm{NO}_{2} \\
\Sigma \mathrm{NO}_{y} \\
\Sigma \mathrm{NO}_{y}-\mathrm{HNO}_{3}\end{array}$ & $\begin{array}{l}\mathrm{NO} / \mathrm{O}_{3} \text { chemi- } \\
\text { luminescence } \\
\text { detectors Pho- } \\
\text { tochemical } \\
\text { convertor + } \\
\text { above } \\
\text { Gold tube/CO } \\
\text { converter + } \\
\text { above } \\
\text { Gold tube con- } \\
\text { vertor and de- } \\
\text { nuder }\end{array}$ & $\sqrt{ } \mathrm{BA}$ & & $10 \mathrm{~min}$ & $\begin{array}{l}3 \text { pptv for } \\
\mathrm{NO}, 7 \text { pptv } \\
\text { for } \mathrm{NO}_{2}\end{array}$ & $\begin{array}{l}15 \% \text { for } \mathrm{NO} \\
\text { and } 20 \% \text { for } \\
\mathrm{NO}_{2} \text { at } 50 \mathrm{pptv}\end{array}$ & Pike et al., 2009 \\
\hline $\begin{array}{l}\mathrm{NO} \\
\mathrm{NO}_{2} \\
\Sigma \mathrm{NO}_{y} \\
\Sigma \mathrm{NO}_{\mathrm{y}}-\mathrm{HNO}_{3}\end{array}$ & $\begin{array}{l}\mathrm{NO} / \mathrm{O}_{3} \text { chemi- } \\
\text { luminescence } \\
\text { detectors Pho- } \\
\text { tochemical } \\
\text { convertor } \\
+ \text { above } \\
\text { Gold tube/CO } \\
\text { converter + } \\
\text { above } \\
\text { Gold tube con- } \\
\text { vertor and de- } \\
\text { nuder }\end{array}$ & & $\sqrt{ }$ & $10 \mathrm{~s}$ & $\begin{array}{l}3 \text { pptv for } \\
\mathrm{NO} \text { and } 15 \\
\text { pptv for } \mathrm{NO}_{2}\end{array}$ & $\begin{array}{l}8 \% \text { for } \mathrm{NO} \text { at } \\
1 \mathrm{ppbv} \text { and } 9 \% \\
\text { for } \mathrm{NO}_{2} \text { at } \\
1 \mathrm{ppbv}\end{array}$ & $\begin{array}{l}\text { (Brough et al., } \\
\text { 2003; Stewart, } \\
\text { et al., 2008) }\end{array}$ \\
\hline $\mathrm{NO}_{2}$ & $\begin{array}{l}\text { Laser-induced } \\
\text { fluorescence } \\
\text { (LIF) }\end{array}$ & $\sqrt{ } \mathrm{BA}$ & & $1 \mathrm{~Hz}$ & $3.6 \mathrm{pptv} / 60 \mathrm{~s}$ & $20 \%$ & $\begin{array}{l}\text { (Dari- } \\
\text { Salisburgo } \\
\text { et al., 2009) }\end{array}$ \\
\hline$\Sigma \mathrm{PNs}$ & $\begin{array}{l}\text { Thermal- } \\
\text { dissociation } \\
\text { LIF }\end{array}$ & $\sqrt{ } \mathrm{BA}$ & & $1 \mathrm{~Hz}$ & $13 \mathrm{pptv} / 60 \mathrm{~s}$ & $40 \%$ & $\begin{array}{l}\text { (Dari- } \\
\text { Salisburgo } \\
\text { et al., 2010) }\end{array}$ \\
\hline$\Sigma$ ANs & $\begin{array}{l}\text { Thermal- } \\
\text { dissociation } \\
\text { LIF }\end{array}$ & $\sqrt{ } \mathrm{BA}$ & & $1 \mathrm{~Hz}$ & $13 \mathrm{pptv} / 60 \mathrm{~s}$ & $40 \%$ & $\begin{array}{l}\text { (Aruffo et al., } \\
2010 \text { ) }\end{array}$ \\
\hline $\mathrm{NO}_{2}$ flux & $\begin{array}{l}\text { LIF-Eddy } \\
\text { covariance }\end{array}$ & $\sqrt{ } \mathrm{BA}$ & & $10 \mathrm{~Hz}$ & $11 \mathrm{pptv} / 60 \mathrm{~s}$ & $20 \%$ & $\begin{array}{l}\text { (Dari- } \\
\text { Salisburgo } \\
\text { et al., 2010) }\end{array}$ \\
\hline
\end{tabular}


Table 1. Continued.

\begin{tabular}{|c|c|c|c|c|c|c|c|}
\hline Species & $\begin{array}{l}\text { Method/ } \\
\text { Analytical } \\
\text { Technique }\end{array}$ & Ground $\dagger$ & Air & $\begin{array}{l}\text { Temporal } \\
\text { Resolution }\end{array}$ & $\begin{array}{l}\text { Detection } \\
\text { Limit }\end{array}$ & $\begin{array}{l}\text { Measurement } \\
\text { Uncertainty }\end{array}$ & Reference \\
\hline $\begin{array}{ll}\mathrm{H}_{2} \mathrm{O}_{2}, & \Sigma \\
\mathrm{ROOH} & \end{array}$ & $\begin{array}{l}\text { Dual-channel } \\
\text { fluorometric } \\
\text { detector }\end{array}$ & & $\sqrt{ }$ & $1 \mathrm{~min}$ & $50 \mathrm{pptv}$ & $14 \%(1 \sigma)$ & $\begin{array}{l}\text { (Penkett et al., } \\
\text { 1995) }\end{array}$ \\
\hline $\mathrm{H}_{2} \mathrm{O}$ vapour & $\begin{array}{l}\text { Dew point } \\
\text { hygrometer }\end{array}$ & $\sqrt{ } \mathrm{BA}$ & $\sqrt{ }$ & & & & \\
\hline $\mathrm{H}_{2} \mathrm{O}$ flux & $\begin{array}{l}\text { Eddy- } \\
\text { covariance } \\
\text { using UV } \\
\text { Absorption }\end{array}$ & $\sqrt{ } \mathrm{BA}$ & & $\begin{array}{l}30 \mathrm{~min} \text { fluxes } \\
100 \mathrm{~Hz}\end{array}$ & & $\begin{array}{l}8 \% \text { (q- } \\
\text { dependent) }\end{array}$ & $\begin{array}{l}\text { (Coe et al., } \\
1995 \text { ) }\end{array}$ \\
\hline $\mathrm{CH}_{2} \mathrm{O}$ & $\begin{array}{l}\text { Fluorometric } \\
\text { detection } \\
\text { (Hantzsh } \\
\text { reaction) }\end{array}$ & $\sqrt{ } \mathrm{BA}$ & & $1 \mathrm{~min}$ & 100 pptv & $17 \%(2 \sigma)$ & $\begin{array}{l}\text { (Still et al., } \\
2006)\end{array}$ \\
\hline $\begin{array}{l}\text { Speciated alde- } \\
\text { hydes, ketones } \\
\text { and alcohols } \\
\text { NMHC, includ- } \\
\text { ing isoprene } \\
\text { and oxygenates }\end{array}$ & $\begin{array}{l}\text { GC/GC detec- } \\
\text { tion, PTR-MS }\end{array}$ & & $\sqrt{ }$ & $15 \mathrm{~s}$ & $50-120 \mathrm{pptv}$ & $13-16 \%(1 \sigma)$ & \\
\hline $\begin{array}{l}>\text { C7 NMHC } \\
\text { (e.g. terpenes) }\end{array}$ & $\begin{array}{l}\text { Adsorbent } \\
\text { tubes \& } \\
\text { GC/TOF-MS }\end{array}$ & & $\sqrt{ }$ & Variable & $50 \mathrm{pptv}$ & $\pm(5 \%+20$ pptv $)$ & $\begin{array}{l}\text { (Capes et al., } \\
\text { 2009) }\end{array}$ \\
\hline $\mathrm{CO}$ & $\begin{array}{l}\text { Chemilu- } \\
\text { minesence }\end{array}$ & $\sqrt{ } \mathrm{BA}$ & $\sqrt{ }$ & & & & $\begin{array}{l}\text { (Gerbig et al., } \\
\text { 1999) }\end{array}$ \\
\hline PAN & $\begin{array}{l}\text { GC/ECD (elec- } \\
\text { tron capture de- } \\
\text { tection) }\end{array}$ & & $\sqrt{ }$ & $90 \mathrm{~s}$ & $5 \mathrm{pptv}$ & $5 \%$ & $\begin{array}{l}\text { (Whalley et al., } \\
\text { 2004) }\end{array}$ \\
\hline PAN & $\begin{array}{l}\text { GC/ECD (elec- } \\
\text { tron capture de- } \\
\text { tection) }\end{array}$ & $\sqrt{ } \mathrm{BA}$ & & $10 \mathrm{~min}$ & $\begin{array}{l}\text { PAN:15 } \\
\text { pptv, PPAN, } \\
\text { MPAN:25 } \\
\text { pptv }\end{array}$ & $20 \%(2 \sigma)$ & $\begin{array}{l}\text { (Harrison et al., } \\
\text { 2006) }\end{array}$ \\
\hline $\begin{array}{l}\text { Alkyl nitrates, } \\
\text { organic } \mathrm{N}\end{array}$ & $\begin{array}{l}\text { GC and neg- } \\
\text { ative ion CI } \\
\text { GC/MS }\end{array}$ & $\sqrt{ } \mathrm{BA}$ & $\sqrt{ }$ & $\begin{array}{l}\text { G: } 1 \mathrm{~h} \\
\text { A: variable }\end{array}$ & $0.005 \mathrm{pptv}$ & $13 \%(2 \sigma)$ & $\begin{array}{l}\text { (Reeves et al., } \\
\text { 2007; Worton } \\
\text { et al., 2008) }\end{array}$ \\
\hline $\begin{array}{l}\text { Reactive } \\
\text { halocarbons }\end{array}$ & GC/MS & $\sqrt{ } \mathrm{BA}$ & $\sqrt{ }$ & $\begin{array}{l}\mathrm{G}: 1 \mathrm{~h} \\
\text { A: variable }\end{array}$ & $0.005 \mathrm{pptv}$ & $15 \%(2 \sigma)$ & $\begin{array}{l}\text { (Worton et al., } \\
\text { 2008) }\end{array}$ \\
\hline Halocarbons & GC/ECD & $\sqrt{ } \mathrm{BA}$ & & $\sim 15 \min$ & $\sim 0.5 \mathrm{pptv}$ & $5-10 \%$ & $\begin{array}{l}\text { (Gostlow et al., } \\
2009 \text { ) }\end{array}$ \\
\hline
\end{tabular}


Table 1. Continued.

\begin{tabular}{|c|c|c|c|c|c|c|c|}
\hline Species & $\begin{array}{l}\text { Method/ } \\
\text { Analytical } \\
\text { Technique }\end{array}$ & Ground $\dagger$ & Air & $\begin{array}{l}\text { Temporal } \\
\text { Resolution }\end{array}$ & $\begin{array}{l}\text { Detection } \\
\text { Limit }\end{array}$ & $\begin{array}{l}\text { Measurement } \\
\text { Uncertainty }\end{array}$ & Reference \\
\hline $\begin{array}{l}\text { Aerosol } \\
\text { number } \\
\text { concentration }\end{array}$ & $\begin{array}{l}\text { CPCs total } \\
\text { particle number } \\
\text { concentration } \\
(>3 \mathrm{~nm})\end{array}$ & $\sqrt{ } \mathrm{BA}$ & $\sqrt{ }$ & $\begin{array}{l}30 \mathrm{~min} \\
\text { (ground) } \\
1 \mathrm{~s} \text { (aircraft) }\end{array}$ & N/A & N/A & \\
\hline \multirow[t]{2}{*}{$\begin{array}{l}\text { Aerosol size } \\
\text { distribution }\end{array}$} & $\begin{array}{l}\text { SMPS ground: } \\
3<D_{\text {md }}<700 \\
\text { nm Air: } \\
3<D_{\text {md }}<350 \\
\text { nm }\end{array}$ & $\sqrt{ } \mathrm{BA} / \mathrm{IC}$ & $\sqrt{ }$ & $\begin{array}{l}30 \text { min } \\
\text { (ground) } \\
1 \text { min } \\
\text { (aircraft) }\end{array}$ & N/A & N/A & \\
\hline & $\begin{array}{l}\text { Optical and } \\
\text { aerodynamic } \\
\text { particle } \\
\text { counters } \\
(0.3 / 0.1- \\
20 / 3 \mu \mathrm{m})\end{array}$ & $\sqrt{ } \mathrm{BA} / \mathrm{IC}$ & $\sqrt{ }$ & $\begin{array}{l}30 \text { min } \\
\text { (ground) } \\
1 \mathrm{~s} \text { (aircraft) } \\
\text { in-canopy } \\
\text { gradients } \\
(2-32 \mathrm{~m})\end{array}$ & N/A & N/A & \\
\hline $\begin{array}{l}\text { Ultra-fine } \\
\text { particle fluxes }\end{array}$ & $\begin{array}{l}\text { Eddy Correla- } \\
\text { tion } D_{p}>3 \mathrm{~nm} \text {, } \\
>10 \mathrm{~nm} \text {, inde- } \\
\text { pendent CPCs }\end{array}$ & $\sqrt{ } \mathrm{BA}$ & & $\begin{array}{l}\text { Fluxes } \\
30 \text { min. Raw } \\
0.3 \mathrm{~s} .\end{array}$ & $\begin{array}{l}<0.01 \\
\text { particle } / \mathrm{cm}^{3}\end{array}$ & $\begin{array}{l} \pm 10 \% @ \\
3 \times 10^{5} \\
\text { particles } / \mathrm{cm}^{3} .\end{array}$ & $\begin{array}{l}\text { (Buzorius et al., } \\
\text { 1998) }\end{array}$ \\
\hline $\begin{array}{l}\text { Aerosol size } \\
\text { segregated } \\
\text { chemical } \\
\text { composition }\end{array}$ & $\begin{array}{l}\text { Aerodyne } \\
\text { Aerosol Mass } \\
\text { Spectro- } \\
\text { meter ( } 40 \mathrm{~nm}< \\
D_{\mathrm{vad}}< \\
0.8 \mu \mathrm{m}), \\
\text { non-refractory } \\
\mathrm{SO}_{4}^{2-}, \mathrm{NO}_{3}^{-}, \\
\mathrm{NH}_{4}^{+} \text {, organic } \\
\text { species }\end{array}$ & $\sqrt{ } \mathrm{BA}, \mathrm{OP}$ & $\sqrt{ }$ & $\begin{array}{l}30 \mathrm{~min} \\
\text { (ground) } \\
10 \mathrm{~s} \text { (profile) } \\
30 \mathrm{~s} \text { (SLR) }\end{array}$ & $\begin{array}{l}\text { Ground: } 3 \mathrm{ng} \\
\mathrm{m}^{-3}\left(\mathrm{NO}_{3}^{-},\right. \\
\left.\mathrm{SO}_{4}^{2-}\right) \text { and } \\
11 \mathrm{ng} \mathrm{m}^{-3} \\
\left(\mathrm{NH}_{4}^{+}\right), 10 \\
\text { min average, } \\
\text { high res } \\
\text { mode } \\
\text { Air: } 3 \mathrm{ng} \\
\mathrm{m}^{-3}\left(\mathrm{NO}_{3}^{-},\right. \\
\left.\mathrm{SO}_{4}^{2-}\right) \text { and } \\
30 \mathrm{ng} \mathrm{m}^{3} \\
\left(\mathrm{NH}_{4}^{+}\right) 30 \mathrm{~s} \\
\text { average }\end{array}$ & $15 \%$ & $\begin{array}{l}\text { Ground: } \\
\text { (Aiken et al., } \\
\text { 2008; Cana- } \\
\text { garatna et al., } \\
\text { 2007) } \\
\text { Aircraft: } \\
\text { (Crosier et al., } \\
\text { 2007) }\end{array}$ \\
\hline $\begin{array}{l}\text { Size segregated } \\
\text { chemically spe- } \\
\text { ciated aerosol } \\
\text { mass fluxes }\end{array}$ & $\begin{array}{l}\text { Eddy- } \\
\text { covariance } \\
\text { using Aero- } \\
\text { dyne Aerosol } \\
\text { Mass Spec- } \\
\text { trometer. (40 } \\
<D_{\text {vad }}<800 \\
\mathrm{~nm} \text { ), non- } \\
\text { refractory } \\
\mathrm{SO}_{4}^{2-} \text {, } \mathrm{NO}_{3}^{-} \text {, } \\
\mathrm{NH}_{4}^{+} \text {, organic } \\
\text { species }\end{array}$ & $\sqrt{ } \mathrm{BA}, \mathrm{OP}$ & & $\begin{array}{l}\text { 30-min } \\
\text { fluxes }\end{array}$ & $\begin{array}{l}10 \mathrm{ng} \mathrm{m}^{-2} \\
\mathrm{~s}^{-1}\end{array}$ & $\begin{array}{l} \pm 10 \mathrm{ng} \mathrm{m}^{-2} \\
\mathrm{~s}^{-1}\end{array}$ & $\begin{array}{l}\text { (Nemitz et al., } \\
\text { 2008) }\end{array}$ \\
\hline
\end{tabular}


Table 1. Continued.

\begin{tabular}{|c|c|c|c|c|c|c|c|}
\hline Species & $\begin{array}{l}\text { Method/ } \\
\text { Analytical } \\
\text { Technique }\end{array}$ & Ground $\dagger$ & Air & $\begin{array}{l}\text { Temporal } \\
\text { Resolution }\end{array}$ & $\begin{array}{l}\text { Detection } \\
\text { Limit }\end{array}$ & $\begin{array}{l}\text { Measurement } \\
\text { Uncertainty }\end{array}$ & Reference \\
\hline $\begin{array}{l}\text { Genetic } \\
\text { bioaerosol }\end{array}$ & $\begin{array}{l}\text { Genetic analy- } \\
\text { sis of filters }\end{array}$ & $\sqrt{ } \mathrm{BA}, \mathrm{IC}, \mathrm{OP}$ & & $12-\mathrm{h}$ & & & \\
\hline $\begin{array}{l}\text { Cloud liquid } \\
\text { water }\end{array}$ & $\begin{array}{l}\text { Gerber PVM- } \\
100\end{array}$ & & $\sqrt{ }$ & $0.1 \mathrm{~s}$ & $0.005 \mathrm{~g} \mathrm{~m}^{-3}$ & & (Gerber, 1991) \\
\hline $\begin{array}{l}\text { Aerosol Scat- } \\
\text { tering proper- } \\
\text { ties }\end{array}$ & $\begin{array}{l}3 \text { wavelength } \\
\text { nephelometer }\end{array}$ & & $\sqrt{ }$ & & $2 \mathrm{~mm}^{-1}$ & $5 \%$ & $\begin{array}{l}\text { (Osborne et al., } \\
\text { 2008) }\end{array}$ \\
\hline $\begin{array}{l}\text { Aerosol hygro- } \\
\text { scopicity }\end{array}$ & $\begin{array}{l}\text { Hygroscopic } \\
\text { Tandem Differ- } \\
\text { ential Mobility } \\
\text { Analyser }\end{array}$ & $\sqrt{ } \mathrm{BA}$ & & $1 \mathrm{~h}$ & & & $\begin{array}{l}\text { (Gysel et al., } \\
\text { 2007) }\end{array}$ \\
\hline $\begin{array}{l}\text { Meteorological } \\
\text { parameters } \\
\text { (Wind speed } \\
\text { \& direction, } \\
\text { solar radiation, } \\
\text { PAR, precipita- } \\
\text { tion, wetness, } \\
\text { pressure, tem- } \\
\text { perature, RH, } \\
\text { turbulence, sen- } \\
\text { sible heat flux) }\end{array}$ & $\begin{array}{l}\text { Standard mete- } \\
\text { orological sen- } \\
\text { sors (aspirated } \\
\text { thermocouples, } \\
\text { Vaisala WXT) }\end{array}$ & $\sqrt{ } \mathrm{BA}, \mathrm{IC}, \mathrm{OP}$ & & $\begin{array}{l}30 \text { min } \\
\text { temperature } \\
\text { gradients }\end{array}$ & & & \\
\hline $\begin{array}{l}\text { Boundary } \\
\text { Layer Height }\end{array}$ & LIDAR & $\sqrt{\mathrm{BA}}$ & & $1 \mathrm{~s}$ & $20-60 \mathrm{~m}$ & & $\begin{array}{l}\text { (Pearson et al., } \\
\text { 2009) }\end{array}$ \\
\hline
\end{tabular}

$\dagger-\mathrm{BA}=$ Bukti Atur site; IC=In-canopy site; OP=oil-palm site (OP3-II).

of variability in the tropospheric $\mathrm{NO}_{2}$ columns (Martin et al., 2002, 2003).

Figure $6 \mathrm{a}$ and $\mathrm{b}$ shows $\mathrm{HCHO}$ and $\mathrm{NO}_{2}$ column distributions over Borneo during April-July 2008, quantified using UV spectroscopic measurements taken by the nadir-viewing data from SCIAMACHY (HCHO; De Smedt et al., 2008) and OMI $\left(\mathrm{NO}_{2}\right.$; ATBD-OMI-02) instruments respectively. $\mathrm{NO}_{2}$ is a strong absorber at UV wavelengths and, because the OMI instrument provides daily coverage, the $\mathrm{NO}_{2}$ column data can be averaged onto a fine resolution grid of $1.0^{\circ} \times 1.0^{\circ}$ (longitude $\times$ latitude). In contrast, we average the HCHO columns onto a coarser $2.5^{\circ} \times 2.0^{\circ}$ grid (to improve the signal-to-noise ratio), since it is a much weaker absorber and because global coverage is only achieved by SCIAMACHY at the Equator every 6 days. Scenes with fractional cloud coverage $>40 \%$ are excluded from our analysis.

Figure $6 \mathrm{~b}$ shows that $\mathrm{NO}_{2}$ measurements from OMI identify anthropogenic $\mathrm{NO}_{\mathrm{x}}$ signals from Bangkok, Jakarta, Surabaya and Singapore. Enhanced HCHO also seen over these cities indicates intense photochemical activity, likely associated with polluted conditions. Over Indochina in April, elevated $\mathrm{HCHO}$ and $\mathrm{NO}_{2}$ columns are loosely correlated 
Table 2. Overview of aircraft flights made in OP3.

\begin{tabular}{|c|c|c|c|}
\hline Flight & Flight description & Date in 2008 & $\begin{array}{l}\text { Take off- } \\
\text { Landing Times } \\
\text { (UTC) }\end{array}$ \\
\hline B384 & $\begin{array}{l}\text { Survey flight (Bukit Atur, oil palm, SW of Kota } \\
\text { Kinabalu) }\end{array}$ & 9 July & $02: 13-05: 32$ \\
\hline B385 & $\begin{array}{l}\text { Bukit Atur (ground sampling site) and SW of Kota } \\
\text { Kinabalu }\end{array}$ & 10 July & $\begin{array}{l}01: 10-05: 04 \\
07: 04-10: 41\end{array}$ \\
\hline B386 & Bukit Atur & 12 July & $\begin{array}{l}01: 02-04: 48 \\
06: 10-9: 59\end{array}$ \\
\hline B387 & $\begin{array}{l}\text { Maliau Basin, } \mathrm{S} \text { of Sabah. } \\
\mathrm{N} \text { of Bukit Atur and } \mathrm{W} \text { of oil palm plantation }\end{array}$ & 13 July & $\begin{array}{l}00: 54-06: 01 \\
06: 30-10: 10\end{array}$ \\
\hline B388 & Bukit Atur and oil palm & 15 July & $\begin{array}{l}00: 54-04: 43 \\
06: 01-09: 33\end{array}$ \\
\hline B389 & Ocean flight SE of Sabah & 16 July & $\begin{array}{l}00: 49-04: 31 \\
05: 39-09: 40\end{array}$ \\
\hline B390 & CASCADE (Marine flight, W and NW of Sabah) & 18 July & $22: 57-03: 14$ \\
\hline B391 & SW of Bukit Atur, oil palm & 19 July & $\begin{array}{l}00: 54-05: 06 \\
06: 15-09: 48\end{array}$ \\
\hline B392 & Mainly oil palm plantation & 21 July & $\begin{array}{l}00: 48-04: 46 \\
05: 53-09: 46\end{array}$ \\
\hline B393 & Ocean flight (NW and SE of Sabah) & 22 July & $00: 43-05: 10$ \\
\hline
\end{tabular}

Local Time is $(\mathrm{UTC}+8)$.

with AATSR fire-counts (not shown) (Arino et al., 2005) and hence are probably due to fire emissions (Fu et al., 2007). During May, enhanced HCHO columns over the Gulf of Thailand may reflect outflow from fires occurring in Sumatra and from the Sibu area of eastern Borneo. However, during the OP3 campaigns, fire activity over Malaysian Borneo was minimal and the levels of $\mathrm{HCHO}$ and $\mathrm{NO}_{2}$ were generally low, with their column amounts typically approaching their background values of $\sim 5 \times 10^{15}$ molecules $\mathrm{cm}^{-2}$ and $\sim 5 \times 10^{14}$ molecules $\mathrm{cm}^{-2}$, respectively. These low values also suggest that during the OP3 measurement period the HCHO source from biogenic VOC oxidation was weak. We observe a slight $\mathrm{HCHO}$ enhancement during April over Danum Valley (Fig. 6a), but it is difficult to assign source attribution.

In Fig. $6 \mathrm{c}$ we show the monthly-mean 12-year time series of (continuous) $\mathrm{HCHO}$ and (non-continuous) $\mathrm{NO}_{2}$ columns, retrieved from the GOME and SCIAMACHY instruments (De Smedt et al., 2008; Martin et al., 2002, 2003), together with the total number of fire-counts detected by the
ATSR/AATSR instruments (Arino et al., 2001, 2005). High correlations between $\mathrm{HCHO}$ and $\mathrm{NO}_{2}$ columns and the firecount data suggest that biomass burning over Borneo drives observed variability of these trace gases. We find that anomalously high $\mathrm{HCHO}$ and $\mathrm{NO}_{2}$ columns were due to intense burning periods associated with strong El Nino conditions, indicated here by the Multivariate El Nino Southern Oscillation (ENSO) Index (Wolter and Timlin, 1998), as expected. For example, during 1997/98 an increased number of forest and peat fires during the warmer and drier El Nino conditions (Levine, 1999) led to extremely high trace gas column concentrations.

Our recent analysis shows that spatial correlations between the 12-year $\mathrm{HCHO}$ and $\mathrm{NO}_{2}$ data, and the associated assimilated meteorological data, vegetation activity and firecounts were strongest in southernmost Borneo and not over the Bukit Atur (Danum Valley) region (Barkley et al., 2009). The low trace gas columns observed over this region during 2008 are consistent with our understanding of past variability. 


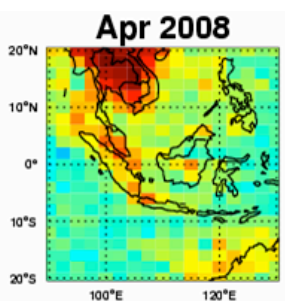

(a)

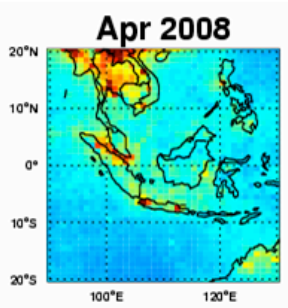

(b)
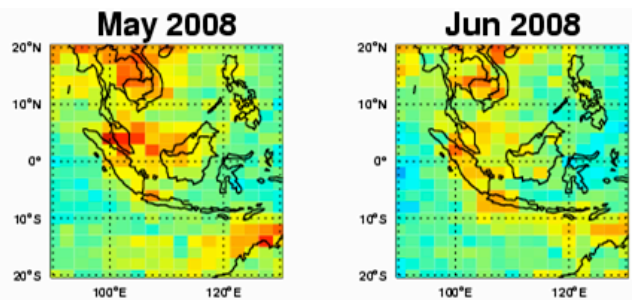

SCIAMACHY HCHO columns $\left[\times 10^{16}\right.$ molecules $\mathrm{cm}^{2}$ ]

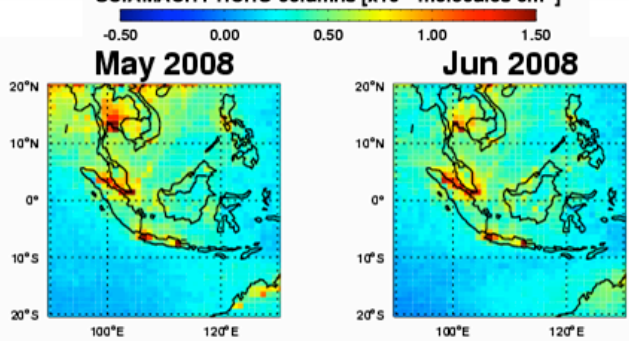

Aura OMI NO${ }_{2}$ columns $\left[\times 10^{15}\right.$ molecules $\mathrm{cm}^{-2}$ ]

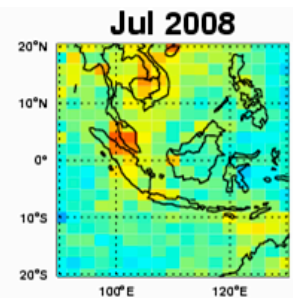

Jul 2008

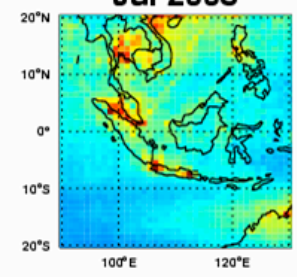

(c)

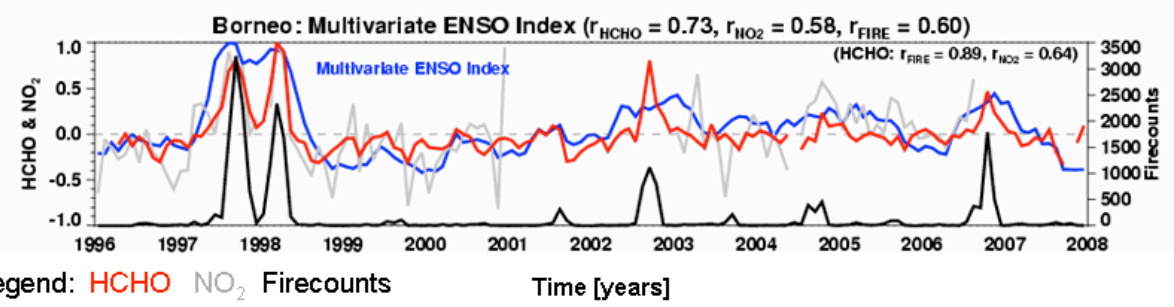

Fig. 6. (a). Monthly averaged SCIAMACHY HCHO columns on a $2.5^{\circ} \times 2.0^{\circ}$ (longitude $\times$ latitude) grid, with cloud coverage $\leq 40 \%$. (b) Monthly averaged $\mathrm{OMI} \mathrm{NO}_{2}$ columns on a $1.0^{\circ} \times 1.0^{\circ}$ grid, with cloud coverage $\leq 40 \%$. (c) The (deseasonalized and normalized) monthlymean time series anomalies over Borneo of $\mathrm{HCHO}$ (red) and $\mathrm{NO}_{2}$ (grey) columns retrieved by the GOME and SCIAMACHY instruments (De Smedt et al., 2008; Martin et al., 2002). The total number of firecounts detected by the ATSR/AATSR instruments (Arino et al., 2001, 2005) (black) and the Multivariate El Nino Southern Oscillation Index (MEI) (Wolter and Timlin, 1998) (blue) are also shown. The correlation of the MEI with the $\mathrm{HCHO}, \mathrm{NO}_{2}$ and firecount timeseries is given in the plot-title; the correlation of the $\mathrm{HCHO}$ timeseries with $\mathrm{NO}_{2}$ and firecounts are shown inset.

\subsection{In-situ observations}

\subsubsection{Airborne measurements}

The in-situ observations made during the OP3 project were centred on sites representative of natural rainforest and oil palm plantations (see above), with aircraft flights over each landscape and over the up- and down-wind oceans (see above). Figure 7 shows aircraft measurements of six species taken on flights near to the Bukit Atur GAW station and the adjacent oil palm plantations. Data are shown from flights (a) below $500 \mathrm{~m}$ above ground level, representative of the boundary layer and (b) above $2500 \mathrm{~m}$, representative of the free troposphere. Also indicated on the plots is the approximate boundary between the natural rainforest and the palm oil plantations.

Within the boundary layer (Fig. 7a), the most striking concentration difference between the rainforest and plantation is for isoprene. Concentrations over the rainforest were typically 1000-3000 pptv, with concentrations over the plantation significantly higher (2-5 times higher; 5000$10000 \mathrm{pptv}$ ). This is consistent with the higher emission rate of isoprene from oil palm trees (Elaeis guineensis) compared to most rainforest tree species (Wilkinson et al., 2006). The plantation landscape also contains associated agro-industrial activities (e.g. road traffic, oil palm processing plants and nitrogenous fertiliser application) and as a result, $\mathrm{NO}_{\mathrm{x}}$ concentrations were also higher compared to the rainforest. However, whereas the higher isoprene concentrations observed tended to be in the west of the oil palm plantation area, higher $\mathrm{NO}_{\mathrm{x}}$ levels were concentrated in the north and east of the plantation area, where the majority of plantation processing plants were observed to be situated. In this area, typical $\mathrm{NO}_{\mathrm{x}}$ levels were $1000-1500 \mathrm{pptv}$, with up to $>2000 \mathrm{pptv}$ observed when flying through or close to the plume of a processing plant. Further south in the plantation area, closer to the boundary with the rainforest, $\mathrm{NO}_{\mathrm{x}}$ was much lower, with levels typically $<300 \mathrm{pptv}$, which is similar to levels that are observed over the rainforest. 

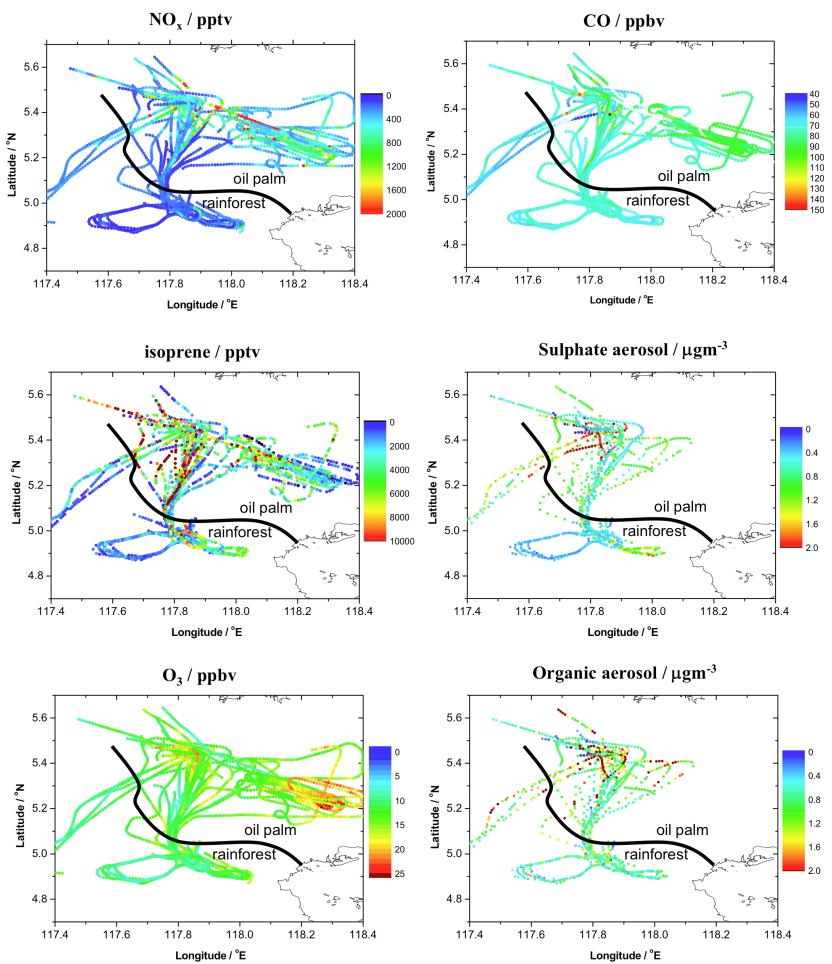

Fig. 7a. Flight track of all data collected from the aircraft below $500 \mathrm{~m}$ (above ground level), coloured by $\mathrm{NO}_{\mathrm{x}}$, isoprene, ozone and organic/sulphate aerosol concentrations.

$\mathrm{CO}$ shows a similar behaviour to $\mathrm{NO}_{\mathrm{x}}$, with higher levels (100-110 ppbv) observed over the northern and eastern plantation area compared to further south and over the rainforest (70-90 ppbv). Levels of sulphate aerosol show little difference between the two land use areas, with both exhibiting aerosol loading of $0.25-1 \mu \mathrm{g} \mathrm{m}^{-3}$.

The result of the increased levels of primary pollutants over the oil palm plantation compared to the rainforest can be seen in the observed ozone, a species that is not directly emitted into the atmosphere but produced photochemically. Ozone was significantly higher (20-25 ppbv) over the eastern area of oil palm plantation, corresponding to the area where $\mathrm{NO}_{\mathrm{x}}$ was generally higher. Over the western area of oil palm plantation, where $\mathrm{NO}_{\mathrm{x}}$ levels are lower but isoprene levels are still high, and over the natural rainforest, where both $\mathrm{NO}_{\mathrm{x}}$ and isoprene are lower, ozone is observed to be $8-15 \mathrm{ppbv}$. This demonstrates the need for increased $\mathrm{NO}_{\mathrm{x}}$ emissions, as well as isoprene, in order for significant extra ozone production to occur (Hewitt et al., 2009). More organic particulate was observed over the oil palm plantation than the rainforest, with average loadings of $0.67 \mu \mathrm{g} \mathrm{m}^{-3}$ and $0.48 \mu \mathrm{g} \mathrm{m}^{-3}$ respectively, which is consistent with our ground based measurements (see below) and could be due to enhanced production of secondary organic material, although primary emissions from the processing plants and human settlements may also be contributing.
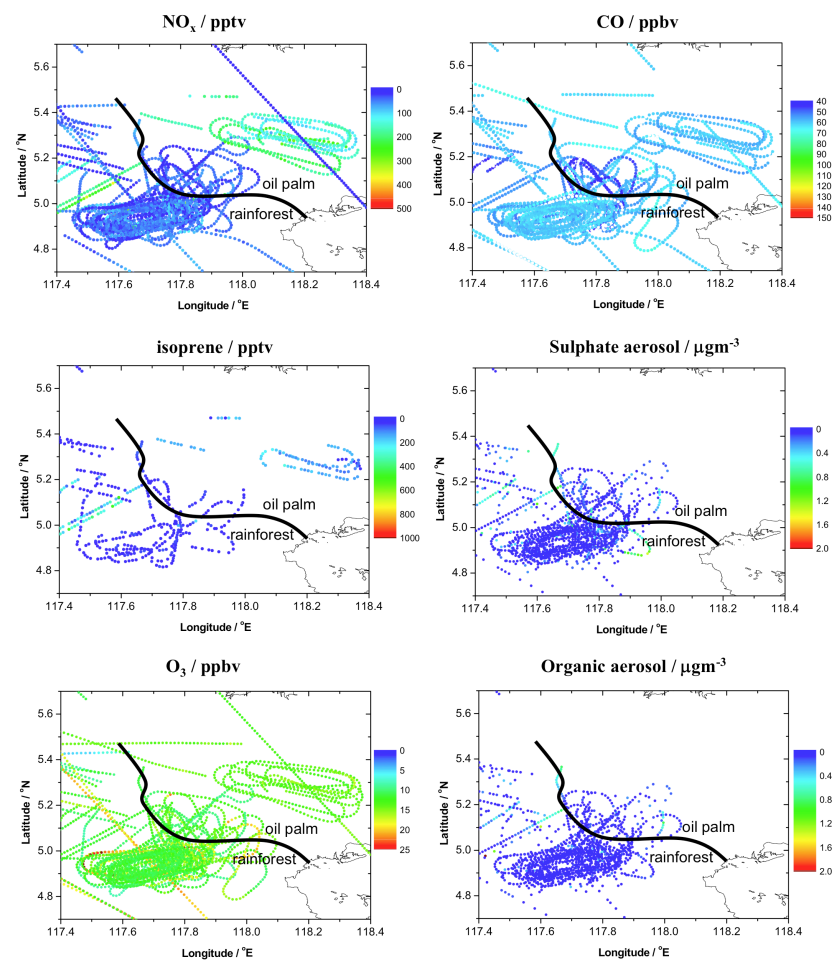

Fig. 7b. Flight track of all data collected from the aircraft above $2500 \mathrm{~m}$ (in the free troposphere), coloured by $\mathrm{NO}_{\mathrm{x}}$, isoprene, ozone and organic/sulphate aerosol content.

In the free troposphere (Fig. 7b), very little isoprene or sulphate aerosol was observed over either the rainforest or plantation and levels of $\mathrm{CO}$ were similar over both areas $(\sim 50$ $60 \mathrm{ppbv}) . \mathrm{NO}_{\mathrm{x}}$ levels were higher in the eastern plantation region (200-300 pptv) compared to the rainforest ( $<50 \mathrm{pptv})$; however this did not lead to increased ozone in the free troposphere over the plantation. This is likely to be due to the very low levels of isoprene and other biogenic VOCs, which do not have a long enough lifetime to escape the boundary layer. Organic aerosol was also very low over both plantation and rainforest in the free troposphere, with an average organic loading of $0.03 \mu \mathrm{g} \mathrm{m}^{-3}$.

As described above, some flights were also undertaken over the ocean, both upwind and downwind of Sabah. Levels of isoprene and $\mathrm{NO}_{\mathrm{x}}$ were very low in both cases and there was no obvious enhancement in ozone in the upwind flights. These data were collected on only two days; however our initial interpretation of this is that Sabah is not a large exporter of primary or processed pollutants to the wider region.

\subsubsection{Bukit Atur measurements}

Data were taken within the rainforest at the Bukit Atur GAW station during two four-week intensive measurement periods (OP3-I and OP3-III). The GAW station has made ozone measurements since January 2007 and these data are shown in 


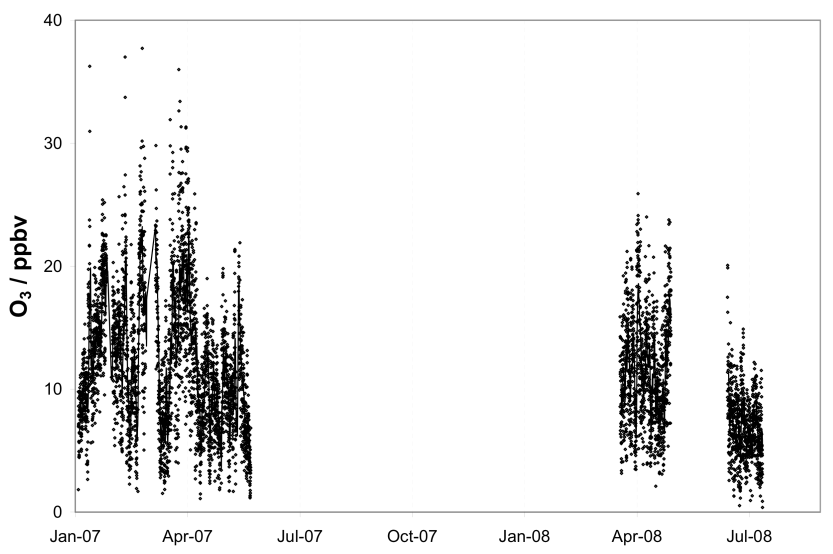

Fig. 8. Ground level ozone concentrations measured at the Bukit Atur GAW site 2007-2008. Data are shown as hourly points with a $24 \mathrm{~h}$ running average.

Fig. 8. Gaps in the data are due to lightning strikes rendering the instrument inoperable. However the plot shows that the levels of ozone observed during the OP3 intensive periods in 2008 are typical for the site.

Campaign daytime (10:00-14:00) average isoprene and total monoterpene fluxes measured at $75 \mathrm{~m}$ on the GAW tower were 1.2 and $0.3 \mathrm{mg} \mathrm{m}^{-2} \mathrm{~h}^{-1}$ respectively during OP3I and 2.1 and $0.49 \mathrm{mg} \mathrm{m}^{-2} \mathrm{~h}^{-1}$ during OP3-III (Langford et al., 2010). These values were comparable to measurements made above regions of the Congo during the EXPRESSO field campaigns (Greenberg et al., 1999; Klinger et al., 1998; Serca et al., 2001) and are somewhat smaller than values reported above regions of the Amazon (Karl et al., 2007; Kuhn et al., 2007). The typical monoterpene to isoprene ratio was between $0.2-0.25$, which is within the upper range of ratios reported in other tropical regions (Guenther et al., 2008). The fluxes of other biogenic VOCs, including methanol, acetone and acetaldehyde, were very much smaller, accounting for just $2 \%$ of the total measured VOC emissions.

Figure 9a shows hourly averaged median diurnal cycles for $\mathrm{NO}, \mathrm{NO}_{2}, \mathrm{O}_{3}$, isoprene and sulphate and organic aerosol mass concentration, taken at Bukit Atur during June and July 2008. Only data from the six days when flights took place over the station were used in the averaging for a direct comparison between the ground-based and aircraft data in the boundary layer and free troposphere over the rainforest. Note that since Bukit Atur is on a ridge, some of the boundary layer data collected by the aircraft over the surrounding forest were at the same or lower altitude (above sea level) as the GAW tower.

For NO, a peak of $\sim 100$ pptv was observed between 07:00 and 08:00 local time, which corresponds to the onset of $\mathrm{NO}_{2}$ photolysis. This was followed by a drop to $\sim 40 \mathrm{pptv}$ by 11:00, with NO then remaining fairly constant throughout the day, until a further drop to $\sim 20 \mathrm{pptv}$ occurred at around 17:00. NO then remained constant throughout the
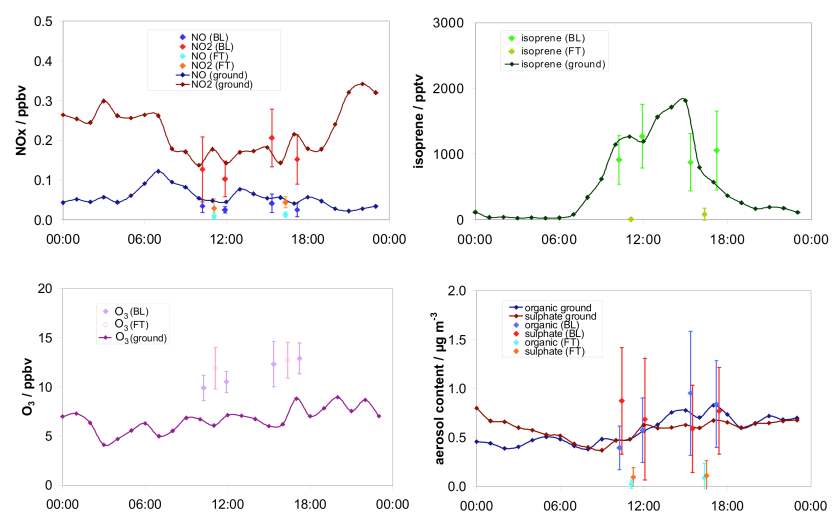

Fig. 9a. Average diurnal concentration profiles for $\mathrm{NO}_{\mathrm{x}}$, isoprene, ozone and organic/sulphate aerosol content for the Bukit Atur ground site and aircraft data taken over surrounding rainforest below $500 \mathrm{~ms}$ above ground level (BL) and in the free troposphere above $2500 \mathrm{~m}$ (FT). The error bars on the aircraft data indicate $1 \sigma$ standard deviations for data taken on the four flights.

night. $\mathrm{NO}_{2}$ exhibits a clear diurnal cycle peaking at around midnight at $300 \mathrm{pptv}$, with a minimum of $\sim 150 \mathrm{pptv}$ in mid-afternoon. These values are comparable to $\mathrm{NO}_{\mathrm{x}}$ measurements made in Amazonia (e.g. Ganzeveld et al., 2008) and over West Africa during the AMMA experiments (e.g. Saunois et al., 2009).

Boundary layer aircraft measurements of $\mathrm{NO}$ and $\mathrm{NO}_{2}$ were in reasonable agreement with the ground based measurements, although airborne NO levels were consistently lower. This, along with the non-zero night-time levels, suggests a local NO source that was diluted throughout the boundary layer. $\mathrm{NO}$ and $\mathrm{NO}_{2}$ levels in the free troposphere were much lower, being $<10$ pptv and $\sim 20$ pptv, respectively. A full description of ground level $\mathrm{NO}_{\mathrm{x}}$ data and discussion of the chemistry is given by Pike et al. (2009) and Pugh et al. (2010b).

Isoprene was the most abundant terpenoid compound observed and showed a clear diurnal cycle with very low ( $<40$ pptv) levels at night and a peak of $\sim 2000$ pptv in mid afternoon. This was caused by the dependence of isoprene emission from trees on temperature and light (Guenther et al., 1993). Boundary layer isoprene measurements from the aircraft were in good agreement with the ground-based Bukit Atur measurements, and free tropospheric isoprene levels were, as expected, very low. Limonene, alpha-pinene and beta-pinene were the most abundant monoterpene compounds observed. Relatively high concentrations of 2-ethyl hexanol and some aldehydes were also seen in samples taken from within the forest canopy in the vicinity of Bukit Atur.

The concentrations of $\mathrm{NO}_{2}$, peroxy nitrate $\left(\mathrm{RO}_{2} \mathrm{NO}_{2}\right)$ and alkyl nitrate $\left(\mathrm{RONO}_{2}\right)$ were measured at Bukit Atur with a laser induced fluorescence system (Aruffo et al., 2009). The diurnal cycle of $\mathrm{RO}_{2} \mathrm{NO}_{2}$ followed the isoprene diurnal cycle, with maximum concentrations in the afternoon. Since 
$\mathrm{RO}_{2} \mathrm{NO}_{2}$ compounds are produced in the oxidation of isoprene and other terpenoids, this result provides evidence for the oxidation of BVOC in the vicinity of the forest canopy. Additional evidence for the active photochemical processing of isoprene is provided by the increase in concentrations of its oxidation products, methyl vinyl ketone and methacrolein, observed in the afternoons.

The concentrations of $\mathrm{HO}_{2}+\mathrm{RO}_{2}$ were measured at Bukit Atur using PEroxy Radical Chemical Amplification (PERCA) technology (Karunaharan et al., 2009) over 20 days in OP3-I and 29 days in OP3-III. Mean midday mixing ratio of $33.5 \pm 3.3$ and $35.5 \pm 3.5$ parts per trillion (pptv) were calculated from the continuous measurements of the sum of the inorganic and organic peroxy radicals from OP3-I and OP3-III respectively. The mean net ozone production rate may then be calculated using:

$$
\begin{aligned}
N\left(\mathrm{O}_{3}\right) & =k_{p}[\mathrm{NO}]\left[\mathrm{HO}_{2}+\mathrm{RO}_{2}\right] \\
& -\left\{f \cdot j\left(\mathrm{O}_{1} \mathrm{D}\right)+k_{1}[\mathrm{OH}]+k_{2}\left[\mathrm{HO}_{2}\right]\right\}\left[\mathrm{O}_{3}\right]
\end{aligned}
$$

where $k_{p}$ is a combined rate coefficient for the oxidation of NO to $\mathrm{NO}_{2}$ by all peroxy radicals and $f \cdot j\left(\mathrm{O}_{1} \mathrm{D}\right)\left[\mathrm{O}_{3}\right]$ represents the fraction of ozone photolysed to yield $\left.\mathrm{O}_{1} \mathrm{D}\right)$ atoms and then $\mathrm{OH}$ (with $f$ being the proportion of $\mathrm{O}\left({ }_{1} \mathrm{D}\right)$ atoms which react with $\mathrm{H}_{2} \mathrm{O}$ to give $\mathrm{OH}$ rather than being collisionally deactivated). The rate constants $k_{1}$ and $k_{2}$ are from the ozone loss reactions:

$$
\begin{aligned}
& \mathrm{OH}+\mathrm{O}_{3}=\mathrm{HO}_{2}+\mathrm{O}_{2} \\
& \mathrm{HO}_{2}+\mathrm{O}_{3}=\mathrm{OH}+2 \mathrm{O}_{2}
\end{aligned}
$$

The net formation of ozone was found to be strongly dependent on the concentration of isoprene, having a linear sensitivity (e.g. $d \ln \left(P\left(\mathrm{O}_{3}\right)\right) / d \ln ($ isoprene $\left.)=1\right)$ ). Since $\mathrm{HO}_{2}+\mathrm{RO}_{2}$ are produced during the oxidation of isoprene and other terpenoids, taken together with the observations of MVK and methacrolein formation, this result provides evidence for the oxidation of BVOC in the vicinity of the forest canopy and of the role of isoprene in the formation of ozone in the nearcanopy environment during the daytime.

Evidence of BVOC reactivity also comes from differential optical absorption spectroscopy (DOAS) measurements. DOAS was used at Bukit Atur to make the first measurements in a tropical rainforest of glyoxal (CHOCHO), one of the major products of isoprene oxidation by $\mathrm{OH}$. Mixing ratios much higher than in other rural locations were observed, with a maximum of $1.6 \mathrm{ppbv}$ and average around $600 \mathrm{pptv}$. $\mathrm{HCHO}$ was also measured, peaking at $4.5 \mathrm{ppbv}$ and with an average around 3 ppbv. Both species were closely correlated to isoprene (which should be their major source in this environment), and peaked in the early afternoon. A box model indicates that the production efficiency of glyoxal from isoprene is $\sim 10 \%$. Although glyoxal was significantly scavenged during rain showers, it is mostly removed by reaction with $\mathrm{OH}$ and photolysis (lifetime at midday $\sim 2.3 \mathrm{~h}$ ) rather than uptake on background aerosol in this environment, because, as discussed earlier, aerosol mass loadings were low.

There was a slight diurnal trend in ground-based $\mathrm{O}_{3}$ measurements, with levels of $\sim 5 \mathrm{ppbv}$ at $06: 00$, rising to $\sim 8 \mathrm{ppbv}$ in mid afternoon. Note that the very small amplitude in the diurnal pattern is typical of a hill-top site (Fowler et al., 2008) and contrasts with that observed at the in-canopy site and over the low-lying palm plantations (see below). These values are comparable to above canopy measurements made above regions of Amazonia (Karl et al., 2007). Boundary layer $\mathrm{O}_{3}$ levels also show an increase throughout the day, but at higher levels (10-13 ppbv), probably because the boundary layer $\mathrm{O}_{3}$ is less depleted by deposition to the canopy (Matsuda et al., 2006; Cros et al., 2000). These boundary layer values are slightly less than those measured over Amazonia and West Africa during the GABRIEL and AMMA campaigns (Ganzveld et al., 2008; Saunois et al., 2009). There is little vertical structure in the $\mathrm{O}_{3}$ mixing ratio at higher heights above the rainforest, with levels in the free troposphere being very similar to those in the boundary layer. Ozone vertical profiles were therefore more like those observed during the monsoon over northern Australia, than those observed during monsoon break periods (Heyes et al., 2009).

Concentrations of $\mathrm{OH}$ display a clear diurnal cycle, peaking at solar noon, with significant concentrations observed: up to $8.7 \times 10^{6}$ molecules $\mathrm{cm}^{-3}$ (60 min average) was recorded on one day. During the GABRIEL campaign above Amazonia, average midday boundary layer $\mathrm{OH}$ concentrations were $5.75 \times 10^{6}$ molecules $\mathrm{cm}^{-3}$ (Martinez et al., 2008). Although $j\left(\mathrm{O}^{1} \mathrm{D}\right)$ levels and humidity were high, the relatively low $\mathrm{O}_{3}$ concentrations limit primary $\mathrm{OH}$ production. The measured $\mathrm{OH}$ reactivity was very high, up to $87 \mathrm{~s}^{-1}$ (corresponding to a lifetime for $\mathrm{OH}$ approaching $10 \mathrm{~ms}$ ). According to the measurements of reactive hydrocarbons (above), this reactivity is dominated by reaction with isoprene, indicating that significant $\mathrm{OH}$ sources must be present - in addition to primary production following ozone photolysis - in order to maintain the elevated levels of $\mathrm{OH}$ levels recorded (Whalley et al., 2010b). The $\mathrm{OH}$ diurnal profile was asymmetric (i.e., lower post-noon than the equivalent time prenoon). This may be due to its main measured sink, isoprene, being present at higher concentrations in the afternoon, but the asymmetric profile could also result from other factors that have yet to be identified. $\mathrm{OH}$ concentrations up to $6 \times 10^{5}$ molecule $\mathrm{cm}^{-3}$ (60 min average) were also recorded on several nights, consistent with measurements by other groups in forested environments (Tan et al., 2001), with some residual $\mathrm{OH}$ observed after $j\left(\mathrm{O}^{1} \mathrm{D}\right)$ had dropped to zero. Measured concentrations of $\mathrm{HO}_{2}$ and the sum of $\mathrm{HO}_{2}+\mathrm{RO}_{2}$ displayed similar profiles.

Boundary layer $\mathrm{OH}$ concentrations recorded on the aircraft over Bukit Atur were similar to those measured at ground level, whereas aircraft $\mathrm{HO}_{2}$ concentrations were higher than measured at the ground, a reflection of the elevated NO levels 
at the surface. A full description of the $\mathrm{OH}, \mathrm{HO}_{2}$ and $\mathrm{OH}$ reactivity measurements is given in Whalley et al. (2009, 2010a) and Edwards et al. (2009, 2010).

The non-refractory sub-micrometre aerosol as measured by aerosol mass spectrometry at the Bukit Atur ground site was mostly composed of organics, sulphate, and ammonium, with low levels of nitrate and chloride. Average loadings were $0.74,0.61,0.21,0.04$ and $0.01 \mu \mathrm{g} \mathrm{m}^{-3}$, respectively, giving a total aerosol loading of $1.61 \mathrm{\mu g} \mathrm{m}^{-3}$. The sulphate was observed to be largely neutralised to ammonium sulphate. Organics showed a diurnal profile peaking around 21:00. The ensemble organic spectrum had a $m / z=44$ to total organics ratio of $0.15 \%$, with prominent peaks at $m / z=55$ and 53, features which would be considered typical of a partially oxygenated organic aerosol (e.g., Hallquist et al., 2009). The sulphate showed no distinct diurnal profile, suggesting it was regional in nature, potentially from the anthropogenic background, volcanoes or oxidised dimethyl sulphate originating from marine phytoplankton (Dacey and Wakeham, 1986). Of all the gas and aerosol species measured at Bukit Atur, sulphate aerosol most clearly showed the influence of regionalscale transport rather than local sources and sinks. Both nitrate and chloride showed strong nocturnal peaks that could be caused by partitioning from the gas phase as the temperature falls and humidity increases at night.

Slightly larger aerosol concentrations were measured from the aircraft than the ground, both over the rainforest and oil palm, with mean total loadings in the boundary layer of 1.66 and $1.78 \mathrm{\mu g} \mathrm{m}^{-3}$, respectively. Aerosol loadings were much lower in the free troposphere, with an average total of $0.22 \mu \mathrm{g} \mathrm{m}^{-3}$. Free-tropospheric aerosol showed a larger sulphate contribution of $69 \%$, compared with $43 \%$ in the boundary layer.

The water uptake ability of the atmospheric aerosol at Bukit Atur was probed in both the sub- and super-saturated regimes with the use of a Hygroscopicity Tandem Differential Mobility Analyser (HTDMA; Cubison et al., 2005; Gysel et al., 2007) and Cloud Condensation Nucleus counter (CCNc; Roberts et al., 2005), respectively. Aerosol hygroscopic growth factors (GF) at $90 \%$ relative humidity (RH) for particles of dry diameter $\left(D_{0}\right)$ from 27 to $217 \mathrm{~nm}$ showed the aerosol to be externally mixed, with a mean growth factor $\left(\mathrm{GF}_{\mathrm{D} 0,90 \%}\right)$ ranging between 1.3 and 1.6 with increasing dry diameter and multiple growth factor modes at each dry size evident. A Scanning Mobility Particle Analyser (SMPS; TSI 3080) was operated upstream of the CCNc, to supply quasi-monodisperse aerosol in the size range 30 to $220 \mathrm{~nm}$. Critical supersaturations $\left(S_{\mathrm{c}}\right)$ derived from fraction activated $\mathrm{CCN}$ spectra, showed a diurnal variation in supersaturation between 0.2 and $0.8 \%$ required to activate particles of a given $D_{0}$, primarily in the dry diameter range 50 to $120 \mathrm{~nm}$.

\subsubsection{Oil palm plantation measurements}

Campaign daytime average (10:00-14:00) isoprene fluxes measured at $15 \mathrm{~m}$ at the Sabahmas oil palm plantation site were $8.6 \mathrm{mg} \mathrm{m}^{-2} \mathrm{~h}^{-1}$ during OP3-II (Misztal et al., 2010a). This is four to seven times greater than the isoprene flux measured at Bukit Atur (see above). The fluxes of other biogenic VOCs, including oxygenated species and more exotic compounds (e.g. estragole: Misztal et al., 2010b), were smaller, contributing $\sim 15 \%$ of the total measured VOC emissions. Net fluxes of monoterpenes were very small $(<1 \%$ of total VOC flux).

Figure $9 \mathrm{~b}$ shows diurnal cycles of data taken at the groundbased site within the Sabahmas oil palm plantation. Species plotted are the same as for the Bukit Atur rainforest ground site, with the exception of $\mathrm{NO}$ and $\mathrm{NO}_{2}$ which were not measured at ground level at Sabahmas. Boundary layer NO and $\mathrm{NO}_{2}$ levels show slightly higher levels compared to the rainforest but the scatter on the data (indicated by the $1 \sigma$ error bars) is much larger. This is indicative of the high $\mathrm{NO}_{\mathrm{x}}$ concentrations observed in certain areas of the plantation landscape, as described above. Free troposphere $\mathrm{NO}$ and $\mathrm{NO}_{2}$ levels over the plantation were again much lower and very similar to those observed over the rainforest.

Ground based isoprene data show a similar diurnal cycle to the rainforest, with near zero levels at night, but with an earlier peak at around 12:00. Isoprene concentrations are much higher in the plantation, with peak daytime levels of $\sim 16000 \mathrm{pptv}$. Isoprene concentrations in the boundary layer over the plantation show a very similar diurnal profile, but with a significantly lower peak of $\sim 5000 \mathrm{pptv}$. The groundbased measurements were made at the top of the oil palm canopy, and hence this difference may be explained by dilution. Also, since the oil palm plantations are generally situated in low-lying, flat land, the aircraft measurements were always made at a significant height above the ground level measurements, unlike at Bukit Atur where the aircraft was able to fly past the base of the GAW tower, located on a hill.

Measurements of $\mathrm{O}_{3}$ at the ground in the plantation exhibit a very different diurnal cycle compared to the rainforest, with near zero night-time concentrations and a peak of $\sim 5$ ppbv at 11:00-12:00. This is followed by a steady decrease throughout the day until $\mathrm{O}_{3}$ reaches close to zero at around 23:00. This general pattern is typical of that for a lowlying site, where $\mathrm{O}_{3}$ lost by reaction with $\mathrm{NO}$ emitted from fertilised plantation soils and deposition at night is not replenished by mixing from aloft, due to the more ready establishment of a stable boundary layer compared with elevated, more windy, sites (Fowler et al., 2008). Data taken in the boundary layer above the plantation show a similar diurnal cycle to the rainforest, with an increase throughout the day to a peak of $\sim 15$ ppbv. This mean value is 2 to 3 ppbv higher than the rainforest levels, but as indicated by the $1 \sigma$ error bars there is scatter in this value, which reflects the variability in the $\mathrm{NO}_{\mathrm{x}}$ and its impact on $\mathrm{O}_{3}$ concentrations. Increased 

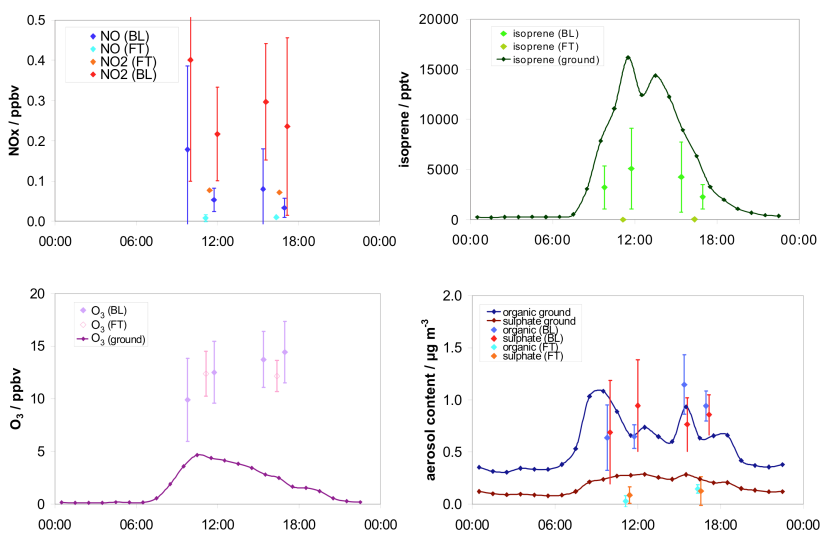

Fig. 9b. Average diurnal profiles for isoprene, ozone and organic/sulphate aerosol content for the oil palm plantation ground site and aircraft data (also with $\mathrm{NO}_{\mathrm{x}}$ ) taken over surrounding oil palm plantations and below $500 \mathrm{~m}$ above ground level (BL) and in the free troposphere above $2500 \mathrm{~m}$ (FT). The error bars on the aircraft data indicate $1 \sigma$ standard deviations for data taken on the four flights.

deposition to the oil palm trees, reaction with $\mathrm{NO}_{\mathrm{x}}$ from local sources, and reaction with isoprene, are three possible contributing factors for the low concentrations and earlier peak of day-time $\mathrm{O}_{3}$ observed on the ground in the plantation.

The total aerosol loading, as measured by the AMS, at the oil palm site was on average $1.6 \mu \mathrm{g} \mathrm{m}^{-3}$, similar to the aerosol loading measured by AMS at the forest site (Nemitz et al., 2009, 2010). The mass was dominated by organic aerosol, which made up on average $65 \%$ of the total mass, with sulphate contributing $20 \%$ of the total mass. The organic aerosol exhibits a clear diurnal trend with two maxima, occurring at approximately 08:00 and 15:00 local time. The ensemble mass spectrum measured at the oil palm resembles the classic oxygenated organic aerosol spectrum (e.g. Hallquist et al., 2009), dominated by mass fragment $m / z$ 44, resulting from carboxylic acid functionality. An examination of mass fragment-specific diurnal profiles indicates that fragments thought to be associated with less oxygenated organic aerosol make a smaller contribution to the afternoon maximum in the organic mass relative to those masses arising from oxygenated fragments, e.g. $m / z 44$.

Sulphate aerosol shows a slightly different trend in mass to the total organics, with a broader maximum during the daytime, although there was large day-to-day variability. Both the nitrate and chloride fractions showed an obvious maximum, coincident with the morning organic aerosol maximum, but both lacked the increase in mass observed in the organic fraction during the afternoon.

\section{Modelling and assessment}

As stated in the introduction above, a fundamental aim of the OP 3 project was to understand the role played by biogenic emissions from the tropical rainforest: what is the nature of the emissions, how are they transformed and how do these processes then impact local, regional and hemispheric scale atmospheric chemistry; what will be the implications of global change for these processes and their impacts?

To address these questions, the OP3 project employed a range of models, varying in their spatial scale of interest and chemical complexity, as shown in Table 3. Thus, a model of transport and chemistry through and above the forest canopy has been used to address the canopy measurements, to study vertical transport of gas and aerosol species, their emission and deposition, and chemical transformation (Ryder et al., 2009). A range of chemical box models (some run along air parcel trajectories) has been employed to study local chemistry, addressing both the ground based and aircraft measurements. These very detailed models aim to inform the chemical mechanisms used in our regional and global models that, in turn, are used to study the impact of the biogenic emissions at the regional and wider scales. They are also used to investigate transport and variability.

Understanding the fast photochemistry processes (mainly involving $\mathrm{HO}_{\mathrm{x}}$ and $\mathrm{RO}_{\mathrm{x}}$ ) is carried out using constrained box models. These use the observed concentrations of species providing $\mathrm{HO}_{\mathrm{x}}$ sources, sinks and inter-conversion pathways, to calculate the concentrations of $\mathrm{OH}, \mathrm{HO}_{2}$ and other short lived species such as the sum of peroxy radicals $\left(\mathrm{RO}_{2}\right)$, for comparison with observations. Insight from these calculations allows our understanding of the complex organic chemistry occurring in this region to be evaluated, the level of chemical complexity required to capture these processes to be quantified and the impact of the approximations made in the chemical transport models to be evaluated.

The constrained-box-model focus is split between the ground-based and aircraft data. One investigation has used the Master Chemical Mechanism, a near-explicit mechanism that considers the degradation of a suite of hydrocarbons in the atmosphere (Jenkin et al., 2003; Saunders et al., 2003). For both ground and aircraft data, a zero-dimensional box model is constrained with the observed concentrations of longer-lived species such as hydrocarbons, $\mathrm{NO}_{\mathrm{x}}, \mathrm{O}_{3}$, relevant meteorological data and observed photolysis rates; the models predict radical concentrations which can be compared with those measured. The ground-based model is a continuation of a previous modelling framework (e.g. Emmerson et al., 2007) that has been used extensively for similar comparisons (Heard and Pilling, 2003). The aircraft based model uses the Dynamically Simple Model of Atmospheric Chemical Complexity (DSMACC) (Emmerson and Evans, 2009) model and follows previous aircraft based work (Jaegle et al, 2000; Olson et al., 2006). Preliminary results show that for the first (April-May) ground-based campaign, 
Table 3. Modelling tools employed in the OP3 project.

\begin{tabular}{|c|c|c|c|}
\hline Model name & Brief description & Domain & References \\
\hline Canopy model & $\begin{array}{l}\text { 1-D transport and } \\
\text { chemistry }\end{array}$ & $\begin{array}{l}\text { Ground to canopy } \\
\text { top - six levels }\end{array}$ & Ryder et al. (2009) \\
\hline MCM - York & $\begin{array}{l}4500 \text { species, }>12500 \\
\text { reactions. Near-explicit } \\
\text { chemistry; long lived } \\
\text { gases constrained by } \\
\text { observations }\end{array}$ & Ground-based box & Emmerson et al. (2007) \\
\hline $\begin{array}{l}\text { Dynamically Simple } \\
\text { Model of Atmospheric } \\
\text { Chemical Complexity } \\
\text { (DSMACC) }\end{array}$ & $\begin{array}{l}\text { Reduced chemistry; } \\
\text { long lived gases } \\
\text { constrained by } \\
\text { observations }\end{array}$ & $\begin{array}{l}\text { Steady state calcula- } \\
\text { tions along aircraft } \\
\text { flight track }\end{array}$ & $\begin{array}{l}\text { Emmerson and Evans } \\
\text { (2009) }\end{array}$ \\
\hline CiTTyCAT & $\begin{array}{l}200 \text { species, }>800 \\
\text { reactions including } \\
\text { MIM2 isoprene } \\
\text { scheme, Jenkin alpha- } \\
\text { pinene and RACM } \\
\text { limonene }\end{array}$ & $\begin{array}{l}\text { Boundary layer: one } \\
\text { well-mixed layer in } \\
\text { daytime, two at night. }\end{array}$ & Pugh et al. (2010b) \\
\hline $\begin{array}{l}\text { Cambridge p- } \\
\text { TOMCAT chemical } \\
\text { transport model }\end{array}$ & $\begin{array}{l}63 \text { species, } 177 \text { reac- } \\
\text { tions. Simple organic } \\
\text { mechanism, including } \\
\text { isoprene. Separate bro- } \\
\text { mocarbon scheme. }\end{array}$ & $\begin{array}{l}\text { Global. Variable reso- } \\
\text { lution, usually } 3^{\circ} \times 3^{\circ} \\
\text { or } 0.5^{\circ} \times 0.5^{\circ}\end{array}$ & $\begin{array}{l}\text { Cook et al. (2007), } \\
\text { Voulgarakis et al. } \\
(2009)\end{array}$ \\
\hline p-TOMCAT box & As above & Box model & Pike et al. (2009) \\
\hline
\end{tabular}

there is reasonable agreement between modelled and measured $\mathrm{OH}$ on some days, but an under-prediction on others. The $\mathrm{OH}$ loss for the OP3 campaigns was dominated by isoprene chemistry, with smaller contributions from $\gamma t$ terpinene and limonene. $\gamma$-Terpinene becomes much more important at night-time, when isoprene emissions are effectively zero. Modelling of the aircraft data is in a more preliminary state but shows relatively good agreement between the observed and simulated $\mathrm{HO}_{2}$ with poorer agreement for the $\mathrm{OH}$. Whether this reflects issues with making $\mathrm{OH}$ observations from aircraft or with our understanding of the chemistry of isoprene will require further analysis.

Reduced chemical mechanisms, of the kind traditionally employed in global chemical transport models, have also been used in box model mode, to compare against the measured constituent data and to investigate the significance of processes. Thus, the CiTTyCAT model (Wild et al., 1996; Evans et al., 2000; Emmerson et al., 2004; Donovan et al., 2005) has been used to study the chemical regimes probed by the OP3 measurements, both over the rainforest and over palm oil plantations, and to explore how local air quality could be affected should palm oil (a major isoprene-emitter) become an even more dominant crop, replacing the rainforest. Hewitt et al. (2009) show that under these circumstances, what happens to local and regional-scale emissions of $\mathrm{NO}_{\mathrm{x}}$ will be crucial. Without control of $\mathrm{NO}_{\mathrm{x}}$ emissions, the levels of ozone could become unacceptably high, damaging both crop and human health.

We find that CiTTyCAT can represent the broad features of atmospheric composition above a tropical rainforest (Pugh et al., 2010a). In particular, the model can fit daytime $\mathrm{NO}_{\mathrm{x}}$ and $\mathrm{O}_{3}$ chemistry. Ambient concentration measurements have been used to estimate a net NO flux of $5 \mu \mathrm{g} \mathrm{N} \mathrm{m}^{-2} \mathrm{~h}^{-1}$. Deposition of intermediate VOC oxidation products can have a very significant influence on their concentrations and the chemistry of the boundary layer, highlighting the need for further investigation into deposition processes over tropical forests.

Another box model study with a reduced chemical mechanism has focussed on the ground-based measurements of ozone and $\mathrm{NO}_{\mathrm{x}}$ (Pike et al., 2009). This study suggests that the chemical mechanism included in the Cambridge global chemistry transport model, p-TOMCAT (Cook et al., 2007; Voulgarakis et al., 2009a, b) can reproduce the measurements 
if a parametrization of mixing out of and into the boundary layer is included.

The Cambridge p-TOMCAT global model has been used to look at regional-scale chemistry. Figure 10 (Pike et al., 2009) shows surface ozone concentrations from two calculations for April 2008, with the model run at two different spatial resolutions (approx $3^{\circ} \times 3^{\circ}$ and $0.5^{\circ} \times 0.5^{\circ}$, respectively). At the lower resolution (typical of multi-annual integrations), the model significantly overestimates surface ozone over Borneo. Little spatial structure is evident and detailed flight track comparisons (not shown) fail to reproduce much observed structure. In contrast, at the high resolution, the model now captures a significant land - sea difference in surface ozone, driven by deposition over the land. Modelled ozone concentrations are still somewhat higher than observed but are now closer to the observations.

\section{Conclusions}

The overall aim of the OP3 project was to better understand the interactions that exist between SE Asian rainforests, atmospheric composition and, ultimately, the Earth's climate system. In pursuing this aim, an extensive field deployment was carried out in 2008, supported by satellite data retrievals and modelling studies. The field deployment, from April to the end of July 2008, comprised extensive composition and flux measurements within and above the rainforest canopy, along with a more limited set of measurements in and above an oil palm plantation. Composition measurements across most of the state of Sabah in northern Borneo, and out to sea on both windward and leeward sides of the island, were provided by flights of the FAAM BAe 146. Remote sensing of land cover has shown that the chosen sites were representative of large areas of Borneo and the rest of insular SE Asia; long-term meteorological and ozone data gathered as part of the Global Atmosphere Watch programme show that weather and ozone mixing ratios during the time period of the field deployment were typical of the long term record.

Composition measurements show that the rainforest site was not impacted significantly by anthropogenic pollution, and this is confirmed by satellite retrievals of $\mathrm{NO}_{2}$ and $\mathrm{HCHO}$. The dominant modulators of atmospheric chemistry at the rainforest site were therefore emissions of BVOCs and soil emissions of reactive nitrogen oxides. At the observed BVOC: $\mathrm{NO}_{\mathrm{x}}$ volume mixing ratio $(\sim 100 \mathrm{pptv} / \mathrm{pptv})$, current chemical models suggest that daytime maximum $\mathrm{OH}$ concentrations should be ca. $10^{5}$ radicals $\mathrm{cm}^{-3}$, but observed $\mathrm{OH}$ concentrations were an order of magnitude greater than this. We confirm, therefore, previous measurements which suggest that an unexplained source of $\mathrm{OH}$ must exist above tropical forests (Lelieveld et al., 2008) and continue to interrogate the data to find explanations for this.

Marked differences in BVOC and $\mathrm{NO}_{\mathrm{x}}$ concentrations, and in BVOC fluxes, were measured in the rainforest and the
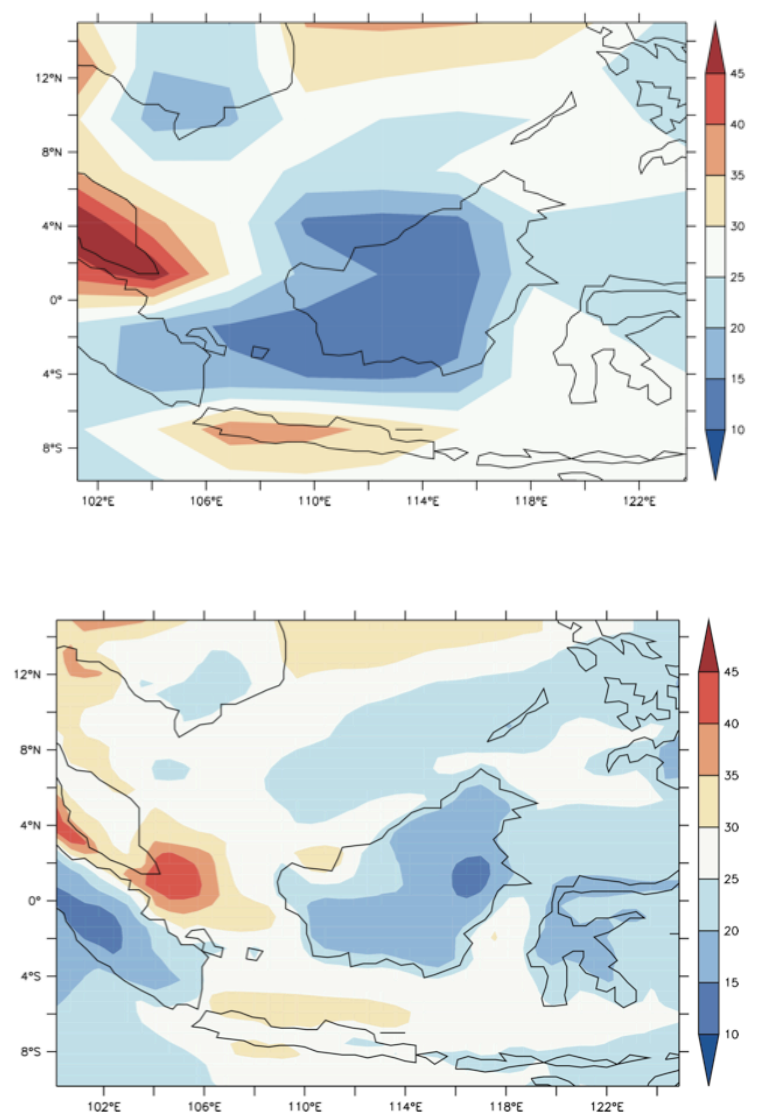

Fig. 10. Mean surface ozone concentrations (ppbv) for April 2008 taken from two integrations of the Cambridge p-TOMCAT chemical transport model. The upper plot shows mean ozone from an integration at $3 \times 3$ degrees horizontal resolution. At this resolution, Borneo is poorly represented and ozone is over estimated. The lower plot shows the same result but when the model is run at $0.5 \times 0.5$ degree horizontal resolution. Ozone concentrations have improved and there is a noticeable land-sea difference consistent with the shape of the island.

oil palm plantation. By an accident of the non-linearity of boundary-layer chemistry, ozone mixing ratios over the two landscapes were similar, although plumes of higher ozone were observed in air impacted by emissions from oil-palm processing plants.

The land-sea mosaic of SE Asia makes it difficult for global atmospheric chemistry-and-transport models run at typical resolution to capture the observed atmospheric composition; runs at higher horizontal resolution provide much better agreement with measurements. A missing $\mathrm{OH}$ source notwithstanding, simple chemistry schemes, as currently implemented in most global models, can match observed ozone and $\mathrm{NO}_{\mathrm{x}}$ diurnal patterns, if a parametrization of mixing out of and into the boundary layer is included.

Overall, the SE Asian rainforest has shown itself to have significant differences from the more intensively studied 
Amazon rainforest - in tree speciation and, hence, in BVOC emission potential, and in the apparent lack of seasonality of emissions. It also exhibits significant differences to the oil palm plantations that are replacing it. A striking similarity, however, is the sustained oxidative capacity of the atmosphere above the rainforest in both SE Asia and Amazonia. Our current inability to adequately explain this limits the confidence we can have in our projections of how atmospheric composition and climate may change in the future.

Acknowledgements. The OP3 project was funded by the UK Natural Environment Research Council (NE/D002117/1). Measurements made during OP3-II were, in part, performed as part of the ACES (Aerosol Coupling in the Earth System; NE/E011179/1) project of the NERC APPRAISE (Aerosol Properties, PRocesses And Influences on the Earth's Climate) programme. We thank the Malaysian and Sabah Governments for their permission to conduct research in Malaysia; the Malaysian Meteorological Department (MMD) for access to the Bukit Atur Global Atmosphere Watch station and their long term ozone record; Leong Chow Peng (formerly of MMD) for her support in the early stages of the project; Wilmar International Ltd (and particularly Simon Siburat and his staff) for access to and considerable logistical support at their PPB Oil Palms Bhd Sabahmas Estate; Waidi Sinun of Yayasan Sabah and his staff and Glen Reynolds of the Royal Society's South East Asian Rainforest Research Programme and his staff for logistical support at the Danum Valley Field Centre; the ground staff, engineers, scientists and pilots of the UK Natural Environment Research Council/UK Meteorological Office's BAe 146-301 large atmospheric research aircraft; the NERC Facility for Ground-based Atmospheric Measurements and Halo Photonics for support with the LIDAR deployment; Phua Mui How of the Universiti Malaysia Sabah for his help with the land cover map; and the rest of the OP3 project team for their individual and collective efforts. Isabelle De Smedt and Michel Van Roozendael (Belgian Institute for Space Aeronomy) provided the GOME and SCIAMACHY $\mathrm{HCHO}$ products and the $\mathrm{OMI} \mathrm{NO}_{2}$ product was downloaded from the NASA Data and Information Services Center. This is paper number 501 of the Royal Society's South East Asian Rainforest Research Programme and is dedicated to the memory of Kate Furneaux, a core member of the OP3 project team, who was killed in a traffic accident while cycling in Leeds on 28 July 2009.

Edited by: W. T. Sturges

\section{References}

Aiken, A. C., Decarlo, P. F., Kroll, J. H., Worsnop, D. R., Huffman, J. A., Docherty, K. S., Ulbrich, I. M., Mohr, C., Kimmel, J. R., Sueper, D., Sun, Y., Zhang, Q., Trimborn, A., Northway, M., Ziemann, P. J., Canagaratna, M. R., Onasch, T. B., Alfarra, M. R., Prevot, A. S. H., Dommen, J., Duplissy, J., Metzger, A., Baltensperger, U., and Jimenez, J. L.: O/C and OM/OC ratios of primary, secondary, and ambient organic aerosols with high-resolution time-of-flight aerosol mass spectrometry, Environ. Sci. Technol., 42, 4478-4485, 2008.

Andreae, M. O., Artaxo, P., Brandao, C., Carswell, F. E., Ciccioli, P., da Costa, A. L., Culf, A. D., Esteves, J. L., Gash,
J. H. C., Grace, J., Kabat, P., Lelieveld, J., Malhi, Y., Manzi, A. O., Meixner, F. X., Nobre, A. D., Nobre, C., Ruivo, M., Silva-Dias, M. A., Stefani, P., Valentini, R., von Jouanne, J., and Waterloo, M. J.: Biogeochemical cycling of carbon, water, energy, trace gases, and aerosols in amazonia: The LBAEUSTACH experiments, J. Geophys. Res.-Atmos., 107, 8066, doi:10.1029/2001JD000524, 2002.

Andreae, M. O., Artaxo, P., Fischer, H., Freitas, S. R., Gregoire, J. M., Hansel, A., Hoor, P., Kormann, R., Krejci, R., Lange, L., Lelieveld, J., Lindinger, W., Longo, K., Peters, W., de Reus, M., Scheeren, B., Dias, M., Strom, J., van Velthoven, P. F. J., and Williams, J.: Transport of biomass burning smoke to the upper troposphere by deep convection in the equatorial region, Geophys. Res. Lett., 28, 951-954, 2001.

Arino, O., Plummer, S., and Defrenne, D.: Fire disturbance: the ten years time series of the ATSR world fire atlas. Proceedings of the MERIS-AATSR workshop 2005, Frascati, Italy, September 2005.

Arino, O., Simon, M., Piccolini, I., and Rosaz, J. M.: The ERS-2 ATSR-2 World Fire Atlas and the ERS-2 ATSR-2 World Burnt Surface Atlas projects. Proceedings of the 8th ISPRS conference on Physical Measurement and Signatures in Remote Sensing, Aussois, 8-12 January 2001.

Aruffo E., Di Carlo P., Dari Salisburgo C., and Giammaria F.: Thermal-dissociation Laser Induced fluorescence (TD-LIF) instrument for NOy speciation: observations of total peroxy nitrate and total alkyl nitrate, Atmos. Chem. Phys. Discuss., in preparation, 2010.

Ashbaugh, L. L., Malm, W. C., and Sadeh, W. Z.: A residence time probability analysis of sulfur concentrations at Grand Canyon National Park, Atmos. Environ., 19, 1263-1270, 1985.

ATBD-OMI-02: OMI Algorithm Theoretical Basis Document Volume IV OMI Trace Gas Algorithms, edited by: Chance, K., Smithsonian Astrophysical Observatory Cambridge, MA, USA, Version 1.0, September 2001.

Aubinet, M., Grelle, A., Ibrom, A., Rannik, U., Moncrieff, J., Foken, T., Kowalski, A. S., Martin, P. H., Berbigier, P., Bernhofer, C., Clement, R., Elbers, J., Granier, A., Grunwald, T., Morgenstern, K., Pilegaard, K., Rebmann, C., Snijders, W., Valentini, R., and Vesala, T.: Estimates of the annual net carbon and water exchange of forests: The EUROFLUX methodology, in Advances in Ecological Research, Vol 30, Academic Press Inc, San Diego, 113-175, 2000.

Avissar, R., Dias, P. L. S., Dias, M., and Nobre, C.: The large-scale biosphere-atmosphere experiment in Amazonia (LBA): Insights and future research needs, J. Geophys. Res.-Atmos., 107, 8086, doi:10.1029/2002JD002704, 2002.

Baillie, I. C.: Soils of the humid tropics, in: The tropical rainforest (Second edition), edited by: Richards P. W., Cambridge University Press, Cambridge, 256-286, 1996.

Barkley, M. P., Palmer, P. I., De Smedt, I., Karl, T., Guenther, A., and Van Roozendael, M.: Regulated large-scale annual shutdown of amazonian isoprene emissions?, Geophys. Res. Lett., 36, L04803, doi:10.1029/2008GL036843, 2009.

Barth, M., McFadden, J., Sun, J., Wiedinmyer, C., Chuang, P., Collins, D., Griffin, R., Hannigan, M., Karl, T., Kim, S.-W., Lasher-Trapp, S., Levis, S., Litvak, M., Mahowald, N., Moore, K., Nandi, S., Nemitz, E., Nenes, A., Potosnak, M., Raymond, T., Smith, J., Still, C., and Stroud, C.: Coupling between land 
ecosystems and the atmospheric hydrologic cycle through biogenic aerosol pathways, B. Am. Meteor. Soc., 86(12), 17381742, 2005.

Bidin, K. and Chappell, N. A.: TECHNICAL NOTE: First evidence of a structured and dynamic spatial pattern of rainfall within a small humid tropical catchment, Hydrol. Earth Syst. Sci., 7, 245253, 2003,

http://www.hydrol-earth-syst-sci.net/7/245/2003/.

Bidin, K. and Chappell, N. A.: Characteristics of rain events at an inland locality in northeastern Borneo, Malaysia, Hydrol. Process., 20, 3835-3850, 2006.

Bohn, B., Corlett, G. K., Gillmann, M., Sanghavi, S., Stange, G., Tensing, E., Vrekoussis, M., Bloss, W. J., Clapp, L. J., Kortner, M., Dorn, H.-P., Monks, P. S., Platt, U., Plass-Dülmer, C., Mihalopoulos, N., Heard, D. E., Clemitshaw, K. C., Meixner, F. X., Prevot, A. S. H., and Schmitt, R.: Photolysis frequency measurement techniques: results of a comparison within the ACCENT project, Atmos. Chem. Phys., 8, 5373-5391, 2008,

http://www.atmos-chem-phys.net/8/5373/2008/.

Brough, N., Reeves, C. E., Penkett, S. A., Stewart, D. J., Dewey, K., Kent, J., Barjat, H., Monks, P. S., Ziereis, H., Stock, P., Huntrieser, H., and Schlager, H.: Intercomparison of aircraft instruments on board the C-130 and Falcon 20 over southern Germany during EXPORT 2000, Atmos. Chem. Phys., 3, 21272138, 2003,

http://www.atmos-chem-phys.net/3/2127/2003/.

Buzorius, G., Rannik, Ü., Mäkelä, J. M., Vesala, T., and Kulmala, M.: Vertical aerosol particle fluxes measured by eddy covariance technique using condensational particle counter, J. Aerosol Sci., 29, 157-171, 1998.

Canagaratna, M. R., Jayne, J. T., Jimenez, J. L., Allan, J. D., Alfarra, M. R., Zhang, Q., Onasch, T. B., Drewnick, F., Coe, H., Middlebrook, A., Delia, A., Williams, L. R., Trimborn, A. M., Northway, M. J., DeCarlo, P. F., Kolb, C. E., Davidovits, P., and Worsnop, D. R.: Chemical and microphysical characterization of ambient aerosols with the Aerodyne aerosol mass spectrometer, Mass Spectrom. Rev., 26, 185-222, 2007.

Capes, G., Murphy, J. G., Reeves, C. E., McQuaid, J. B., Hamilton, J. F., Hopkins, J. R., Crosier, J., Williams, P. I., and Coe, H.: Secondary organic aerosol from biogenic VOCs over West Africa during AMMA, Atmos. Chem. Phys., 9, 3841-3850, 2009, http://www.atmos-chem-phys.net/9/3841/2009/.

Carslaw, N., Creasey, D. J., Harrison, D., Heard, D. E., Hunter, M. C., Jacobs, P. J., Jenkin, M. E., Lee, J. D., Lewis, A. C., Pilling, M. J., Saunders, S. M., and Seakins, P. W.: $\mathrm{OH}$ and $\mathrm{HO}_{2}$ radical chemistry in a forested region of north-western Greece, Atmos. Environ., 35, 4725-4737, 2001.

Chappell, N. A., Sherlock, M., Bidin, K., Macdonald, R., Najman, Y., and Davies, G.: Runoff processes in Southeast Asia: role of soil, regolith and rock type, in: Forest Environments in the Mekong River Basin, edited by: Sawada, H., Araki, M., Chappell, N. A., LaFrankie, J. V., and Shimizu ,A., Springer Verlag, Tokyo, 3-23, 2007.

Chappell, N. A., Bidin, K., and Tych, W.: Modelling rainfall and canopy controls on net-precipitation beneath selectively-logged tropical forest, Plant Ecol., 153, 215-229, 2001.

Coe, H., Gallagher, M. W., Choularton, T. W., and Dore, C.: Canopy Scale Measurements of Stomatal and Cuticular O3 Uptake by Sitka Spruce, Atmos. Environ., 29, 1413-1423, 1995.
Conrad, R.: Compensation Concentration as Critical Variable for Regulating the Flux of Trace Gases between Soil and Atmosphere, Biogeochemistry, 27, 155-170, 1994.

Cook, P. A., Savage, N. H., Turquety, S., Carver, G. D., O’Connor, F. M., Heckel, A., Stewart, D., Whalley, L. K., Parker, A. E., Schlager, H., Singh, H. B., Avery, M. A., Sachse, G. W., Brune, W., Richter, A., Burrows, J. P., Purvis, R., Lewis, A. C., Reeves, C. E., Monks, P. S., Levine, J. G., and Pyle, J. A.: Forest fire plumes over the north Atlantic: p-TOMCAT model simulations with aircraft and satellite measurements from the ITOP/ICARTT campaign, J. Geophys. Res.-Atmos., 112, D10S43, doi:10.1029/2006JD007563, 2007.

Cros, B., Delon, C., Affre, C., Marion, T., Druilhet, A., Perros, P. E., and Lopez, A.: Sources and sinks of ozone in savanna and forest areas during EXPRESSO: Airborne turbulent flux measurements, J. Geophys. Res.-Atmos., 105, 29347-29358, 2000.

Crosier, J., Allan, J. D., Coe, H., Bower, K. N., Formenti, P., and Williams, P. I.: Chemical composition of summertime aerosol in the Po Valley (Italy), northern Adriatic and Black Sea, Q. J. Roy. Meteorol. Soc., 133, 61-75, 2007.

Cubison, M. J., Coe, H., and Gysel, M.: A modified hygroscopic tandem DMA and a data retrieval method based on optimal estimation, J. Aerosol Sci., 36, 846-865, 2005.

Dacey, J. W. H. and Wakeham, S. G.: Oceanic dimethylsulfide - production during zooplankton grazing on phytoplankton, Science, 233, 1314-1316, 1986.

Dari-Salisburgo C., Di Carlo P., Aruffo E., Langford B., Dorsey J., and Giammaria F.: Flux measurements of $\mathrm{NO}_{2}$ in Asian tropical rainforest using laser induced fluorescence and eddy covariance, Atmos. Chem. Phys. Discuss., in preparation 2010.

Dari-Salisburgo, C., Di Carlo, P., Giammaria, F., Kajii, Y., and D'Altorio, A.: Laser induced fluorescence instrument for $\mathrm{NO}_{2}$ measurements: observations at a central Italy background site, Atmos. Environ., 43, 970-977, 2009.

De Smedt, I., Müller, J.-F., Stavrakou, T., van der A, R., Eskes, H., and Van Roozendael, M.: Twelve years of global observations of formaldehyde in the troposphere using GOME and SCIAMACHY sensors, Atmos. Chem. Phys., 8, 4947-4963, 2008, http://www.atmos-chem-phys.net/8/4947/2008/.

Donovan, R. G., Hope, E. S., Owen, S. M., Mackenzie, A. R., and Hewitt, C. N.: Development and Application of an Urban Tree Air Quality Score for Photochemical Pollution Episodes Using the Birmingham, United Kingdom, Area as a Case Study, Environ. Sci. Technol., 39, 6730-6738, 2005.

Dorsey, J. R and Gallagher, M. W.: $\mathrm{NO}_{\mathrm{x}}$ flux measurements from a south east Asian tropical rainforest soil, Atmos. Chem. Phys. Discuss., in preparation, 2010.

Edwards, G. D. and Monks, P. S.: Performance of a singlemonochromator diode array spectroradiometer for the determination of actinic flux and atmospheric photolysis frequencies, J. Geophys. Res., 108, 8546, doi:10.1029/2002JD002844, 2003.

Edwards, P. M., Edwards, P., Whalley, L. K., Heard, D. E., et al.: $\mathrm{OH}$ reactivity measurements in a South-East Asian tropical Rainforest, Atmos. Chem. Phys. Discuss., in preparation, 2010.

Emmerson, K. M., MacKenzie, A. R., Owen, S. M., Evans, M. J., and Shallcross, D. E.: A Lagrangian model with simple primary and secondary aerosol scheme 1: comparison with UK PM10 data, Atmos. Chem. Phys., 4, 2161-2170, 2004, http://www.atmos-chem-phys.net/4/2161/2004/. 
Emmerson, K. M. and Evans, M. J.: Comparison of tropospheric gas-phase chemistry schemes for use within global models, Atmos. Chem. Phys., 9, 1831-1845, 2009, http://www.atmos-chem-phys.net/9/1831/2009/.

Emmerson, K. M., Carslaw, N., Carslaw, D. C., Lee, J. D., McFiggans, G., Bloss, W. J., Gravestock, T., Heard, D. E., Hopkins, J., Ingham, T., Pilling, M. J., Smith, S. C., Jacob, M., and Monks, P. S.: Free radical modelling studies during the UK TORCH Campaign in Summer 2003, Atmos. Chem. Phys., 7, 167-181, 2007, http://www.atmos-chem-phys.net/7/167/2007/.

Emmerson, K. M., Carslaw, N., and Pilling, M. J.: Urban Atmospheric Chemistry during the PUMA Campaign. 2: Radical budgets for $\mathrm{OH}, \mathrm{HO}_{2}$ and $\mathrm{RO}_{2}$, J. Atmos. Chem., 52(2), 165-183, 2005.

European Centre for Medium-Range Weather Forecasts, ECMWF Trajectories: British Atmospheric Data Centre, 2006-2009, online available at: http://badc.nerc.ac.uk/data/ecmwf-trj/, March 2009.

Evans, M. J., Shallcross, D. E., Law, K. S., Wild, J. O. F., Simmonds, P. G., Spain, T. G., Berrisford, P., Methven, J., Lewis, A. C., McQuaid, J. B., Pilling, M. J., Bandy, B. J., Penkett, S. A., And Pyle, J. A.: Evaluation of a Langrangian box model using field measurements from EASE (Eastern Atlantic Summer Experiment) 1996, Atmos. Environ., 34, 3843-3863, 2000.

Farmer, D. K., Wooldridge, P. J., and Cohen, R. C.: Application of thermal-dissociation laser induced fluorescence (TD-LIF) to measurement of $\mathrm{HNO}_{3}$, इalkyl nitrates, $\Sigma$ peroxy nitrates, and $\mathrm{NO}_{2}$ fluxes using eddy covariance, Atmos. Chem. Phys., 6, 3471-3486, 2006,

http://www.atmos-chem-phys.net/6/3471/2006/.

Fleming, Z. L., Monks, P. S., Rickard, A. R., Heard, D. E., Bloss, W. J., Seakins, P. W., Still, T. J., Sommariva, R., Pilling, M. J., Morgan, R., Green, T. J., Brough, N., Mills, G. P., Penkett, S. A., Lewis, A. C., Lee, J. D., Saiz-Lopez, A., and Plane, J. M. C.: Peroxy radical chemistry and the control of ozone photochemistry at Mace Head, Ireland during the summer of 2002, Atmos. Chem. Phys., 6, 2193-2214, 2006,

http://www.atmos-chem-phys.net/6/2193/2006/.

Fowler, D.: Ground-level ozone in the 21 st century: future trends, impacts and policy implications, Royal Society, London, 2008.

Fu, T.-M., Jacob, D. J., Palmer, P. I., Chance, K., Wang, Y. X., Barletta, B., Blake, D. R., Stanton, J. C., and Pilling, M. J.: Space-based formaldehyde measurements as constraints on volatile organic compound emissions in east and south Asia and implications for ozone, J. Geophys. Res., 112, D06312, doi:10.1029/2006JD007853, 2007.

Fueglistaler, S. and Haynes, P. H.: Control of interannual and longer-term variability of stratospheric water vapor, J. Geophys. Res.-Atmos., 110, D24108, doi:10.1029/2005JD006019, 2005.

Ganzeveld, L., Eerdekens, G., Feig, G., Fischer, H., Harder, H., Königstedt, R., Kubistin, D., Martinez, M., Meixner, F. X., Scheeren, H. A., Sinha, V., Taraborrelli, D., Williams, J., ViláGuerau de Arellano, J., and Lelieveld, J.: Surface and boundary layer exchanges of volatile organic compounds, nitrogen oxides and ozone during the GABRIEL campaign, Atmos. Chem. Phys., 8, 6223-6243, 2008,

http://www.atmos-chem-phys.net/8/6223/2008/.

Gerber, H.: Direct Measurement of Suspended Particulate Volume Concentration and Far-Infrared Extinction Coefficient with a Laser-Diffraction Instrument, Appl. Optics, 30, 4824-4831, 1991.

Gerbig, C., Schmitgen, S., Kley, D., Volz-Thomas, A., Dewey, K., and Haaks, D.: An improved fast-response vacuum-UV resonance fluorescence CO instrument, J. Geophys. Res., 104, 16991704, 1999.

Global Forest Resources: Assessment 2005, FAO, Rome, ISBN-925-105481-9, 2006.

Gostlow, B., Robinson, A. D., Harris, N. R. P., O'Brien, L. M., Oram, D. E., Mills, G. P., Newton, H. M., and Pyle, J. A.: $\mu$ Dirac: an autonomous instrument for halocarbon measurements, Atmos. Meas. Tech. Discuss., 2, 2123-2159, 2009,

http://www.atmos-meas-tech-discuss.net/2/2123/2009/.

Gostlow, B., Robinson, A. D., Harris, N. R. P., O’Brien, L. M., Oram, D. E., Mills, G. P., Newton, H. M., and Pyle, J. A.: Dirac: an autonomous instrument for halocarbon measurements, Atmos. Meas. Tech. Discuss., 2, 2123-2159, 2009,

http://www.atmos-meas-tech-discuss.net/2/2123/2009/.

Greenberg, J. P., Guenther, A. B., Madronich, S., Baugh, W., Ginoux, P., Druilhet, A., Delmas, R., and Delon, C.: Biogenic volatile organic compound emissions in central Africa during the experiment for the regional sources and sinks of oxidants (EXPRESSO) biomass burning season, J. Geophys. Res.-Atmos., 104, 30659-30671, 1999.

Guenther, A., Hewitt, C. N., Erickson, D., Fall, R., Geron, C., Graedel, T., Harley, P., Klinger, L., Lerdau, M., McKay, W. A., Pierce, T., Scholes, B., Steinbrecher, R., Tallamraju, R., Taylor, J., and Zimmerman, P.: A global-model of natural volatile organic-compound emissions, J. Geophys. Res.-Atmos., 100, 8873-8892, 1995.

Guenther, A., Karl, T., Harley, P., Wiedinmyer, C., Palmer, P. I., and Geron, C.: Estimates of global terrestrial isoprene emissions using MEGAN (Model of Emissions of Gases and Aerosols from Nature), Atmos. Chem. Phys., 6, 3181-3210, 2006, http://www.atmos-chem-phys.net/6/3181/2006/.

Guenther, A. B., Zimmerman, P. R., Harley, P. C., Monson, R. K., and Fall, R.: Isoprene and monoterpene emission rate variability - model evaluations and sensitivity analyses, J. Geophys. Res.Atmos., 98, 12609-12617, 1993.

Guenther, A., Hewitt, C. N., Karl. T., Harley, P., and Reeves. C.: Biogenic VOC emissions from African American and Asian tropical rainforests, Eos Trans. AGU, 89(53), Fall Meet. Suppl., Abstract A14C-04, 2008.

Güsten, H., Heinrich, G., Schmidt, R. W. H., and Schurath, U.: A Novel Ozone Sensor for Direct Eddy Flux Measurements, paper presented at 7th International Symp of the Commission for Atmospheric Chemistry and Global Pollution : the Chemistry of the Global Atmosphere, Kluwer Academic Publ, Chamrousse, France, 5-11 September, 1990.

Gusten, H. and Heinrich, G.: On-line measurements of ozone surface fluxes .1. methodology and instrumentation, Atmos. Environ., 30, 897-909, 1996.

Gysel, M., Crosier, J., Topping, D. O., Whitehead, J. D., Bower, K. N., Cubison, M. J., Williams, P. I., Flynn, M. J., McFiggans, G. B., and Coe, H.: Closure study between chemical composition and hygroscopic growth of aerosol particles during TORCH2, Atmos. Chem. Phys., 7, 6131-6144, 2007, http://www.atmos-chem-phys.net/7/6131/2007/.

Hallquist, M., Wenger, J. C., Baltensperger, U., Rudich, Y., Simp- 
son, D., Claeys, M., Dommen, J., Donahue, N. M., George, C., Goldstein, A. H., Hamilton, J. F., Herrmann, H., Hoffmann, T., Iinuma, Y., Jang, M., Jenkin, M. E., Jimenez, J. L., Kiendler-Scharr, A., Maenhaut, W., McFiggans, G., Mentel, Th. F., Monod, A., Prévôt, A. S. H., Seinfeld, J. H., Surratt, J. D., Szmigielski, R., and Wildt, J.: The formation, properties and impact of secondary organic aerosol: current and emerging issues, Atmos. Chem. Phys., 9, 5155-5235, 2009,

http://www.atmos-chem-phys.net/9/5155/2009/.

Harrison, R. M., Yin, J., Tilling, R. M., Cai, X., Seakins, P. W., Hopkins, J. R., Lansley, D. L., Lewis, A. C., Hunter, M. C., Heard, D. E., Carpenter, L. J., Creasy, D. J., Lee, J. D., Pilling, M. J., Carslaw, N., Emmerson, K. M., Redington, A., Derwent, R. G., Ryall, D., Mills, G., and Penkett, S. A.: Measurement and modelling of air pollution and atmospheric chemistry in the UK West Midlands conurbation: Overview of the PUMA Consortium project, Sci. Total Environ., 360, 5-25, 2006.

Heard, D. E., Read, K. A., Methven, J., Al-Haider, S., Bloss, W. J., Johnson, G. P., Pilling, M. J., Seakins, P. W., Smith, S. C., Sommariva, R., Stanton, J. C., Still, T. J., Ingham, T., Brooks, B., De Leeuw, G., Jackson, A. V., McQuaid, J. B., Morgan, R., Smith, M. H., Carpenter, L. J., Carslaw, N., Hamilton, J., Hopkins, J. R., Lee, J. D., Lewis, A. C., Purvis, R. M., Wevill, D. J., Brough, N., Green, T., Mills, G., Penkett, S. A., Plane, J. M. C., Saiz-Lopez, A., Worton, D., Monks, P. S., Fleming, Z., Rickard, A. R., Alfarra, M. R., Allan, J. D., Bower, K., Coe, H., Cubison, M., Flynn, M., McFiggans, G., Gallagher, M., Norton, E. G., O’Dowd, C. D., Shillito, J., Topping, D., Vaughan, G., Williams, P., Bitter, M., Ball, S. M., Jones, R. L., Povey, I. M., O’Doherty, S., Simmonds, P. G., Allen, A., Kinnersley, R. P., Beddows, D. C. S., Dall'Osto, M., Harrison, R. M., Donovan, R. J., Heal, M. R., Jennings, S. G., Noone, C., and Spain, G.: The North Atlantic Marine Boundary Layer Experiment (NAMBLEX). Overview of the campaign held at Mace Head, Ireland, in summer 2002, Atmos. Chem. Phys., 6, 2241-2272, 2006,

http://www.atmos-chem-phys.net/6/2241/2006/.

Heard, D. E. and Pilling, M. J.: Measurement of $\mathrm{OH}$ and $\mathrm{HO}_{2}$ in the troposphere, Chem. Rev., 103, 5163-5198, 2003.

Helfter, C., Phillips, G. J., Coyle, M., Di Marco, C. F., Langford, B., Whitehead, J., Dorsey, J. R., Gallagher, M. W., Sei, E. Y., Fowler, D., and Nemitz, E.: Momentum and heat exchange above South East Asian rainforest in complex terrain. Atmos. Chem. Phys. Discuss., in preparation, 2010.

Hewitt, C. N., MacKenzie, A. R., Di Carlo, P., Di Marco, C. F., Dorsey, J. R., Evans, M., Fowler, D., Gallagher, M. W., Hopkins, J. R., Jones, C. E., Langford, B., Lee, J. D., Lewis, A. C., Lim, S. F., McQuaid, J., Misztal, P., Moller, S. J., Monks, P. S., Nemitz, E., Oram, D. E., Owen, S. M., Phillips, G. J., Pugh, T. A. M., Pyle, J. A., Reeves, C. E., Ryder, J., Siong, J., Skiba, U., and Stewart, D. J.: Nitrogen management is essential to prevent tropical oil palm plantations from causing ground-level ozone pollution, P. Natl. Acad. Sci. USA, 106, 18447-18451, 2009.

Ingham, T., Goddard, A., Whalley, L. K., Furneaux, K. L., Edwards, P. M., Seal, C. P., Self, D. E., Johnson, G. P., Read, K. A., Lee, J. D., and Heard, D. E.: A flow-tube based laser-induced fluorescence instrument to measure $\mathrm{OH}$ reactivity in the troposphere, Atmos. Meas. Tech., 2, 465-477, 2009,

http://www.atmos-meas-tech.net/2/465/2009/.

Jaegle, L., Jacob, D. J., Brune, W. H., Faloona, I., Tan, D., Heikes,
B. G., Kondo, Y., Sachse, G. W., Anderson, B., Gregory, G. L., Singh, H. B., Pueschel, R., Ferry, G., Blake, D. R., and Shetter, R. E.: Photochemistry of $\mathrm{HO}_{\mathrm{x}}$ in the upper troposphere at northern midlatitudes, J. Geophys. Res.-Atmos., 105(D3), 3877-3892, 2000.

Jenkin, M. E., Saunders, S. M., Wagner, V., and Pilling, M. J.: Protocol for the development of the Master Chemical Mechanism, MCM v3 (Part B): tropospheric degradation of aromatic volatile organic compounds, Atmos. Chem. Phys., 3, 181-193, 2003, http://www.atmos-chem-phys.net/3/181/2003/.

Kanakidou, M., Seinfeld, J. H., Pandis, S. N., Barnes, I., Dentener, F. J., Facchini, M. C., Van Dingenen, R., Ervens, B., Nenes, A., Nielsen, C. J., Swietlicki, E., Putaud, J. P., Balkanski, Y., Fuzzi, S., Horth, J., Moortgat, G. K., Winterhalter, R., Myhre, C. E. L., Tsigaridis, K., Vignati, E., Stephanou, E. G., and Wilson, J.: Organic aerosol and global climate modelling: a review, Atmos. Chem. Phys., 5, 1053-1123, 2005, http://www.atmos-chem-phys.net/5/1053/2005/.

Kaye, P. H., Stanley, W. R., Hirst, E., Foot, E. V., Baxter, K. L., and Barrington, S. J.: Single particle multichannel bio-aerosol fluorescence sensor, Optics Express, 13(10), 3583-3593, 2005.

Klinger, L. F., Greenberg, J., Guenther, A., Tyndall, G., Zimmerman, P., M'Bangui, M., and Moutsambote, J. M.: Patterns in volatile organic compound emissions along a savanna-rainforest gradient in central Africa, J. Geophys. Res.-Atmos., 103, 14431454, 1998.

Langford, B., Davison, B., Nemitz, E., and Hewitt, C. N.: Mixing ratios and eddy covariance flux measurements of volatile organic compounds from an urban canopy (Manchester, UK), Atmos. Chem. Phys., 9, 1971-1987, 2009, http://www.atmos-chem-phys.net/9/1971/2009/.

Langford, B., Misztal, P. Nemitz, E., Davison, B., Helfter, C., Lee, J., MacKenzie, A. R., and Hewitt, C. N.: Fluxes of volatile organic compounds from a south-east Asian tropical rainforest, Atmos. Chem. Phys. Discuss., in preparation, 2010.

Leff, B., Ramankutty, N., and Foley, J. A.: Geographic distribution of major crops across the world, Global Biogeochem. Cy., 18, GB1009, doi:10.1029/2003GB002108, 2004.

Leigh, R. J., Corlett, G. K., Friess, U., and Monks, P. S.: Concurrent multiaxis differential optical absorption spectroscopy system for the measurement of tropospheric nitrogen dioxide, Appl. Optics, 45, 7504-7518, 2006.

Lelieveld, J., Butler, T. M., Crowley, J. N., Dillon, T. J., Fischer, H., Ganzeveld, L., Harder, H., Lawrence, M. G., Martinez, M., Taraborrelli, D., and Williams, J.: Atmospheric oxidation capacity sustained by a tropical forest, Nature, 452, 737-740, 2008.

Levine, J.: The 1997 Fires in Kalimantan and Sumatra, Indonesia: Gaseous and Particulate Emissions, Geophys. Res. Lett., 26(7), 815-818, 1999.

Lewis, A. C., Evans, M. J., Methven, J., Watson, N., Lee, J. D., Hopkins, J. R., Purvis, R. M., Arnold, S. R., McQuaid, J. B., Whalley, L. K., Pilling, M. J., Heard, D. E., Monks, P. S., Parker, A. E., Reeves, C. E., Oram, D. E., Mills, G., Bandy, B. J., Stewart, D., Coe, H., Williams, P., and Crosier J.: Chemical composition observed over the mid-Atlantic and the detection of pollution signatures far from source regions, J. Geophys. Res.-Atmos., 112, D10S39, doi:10.1029/2006JD007584, 2007.

Lewis, A. C., Hopkins, J. R., Carpenter, L. J., Stanton, J., Read, K. A., and Pilling, M. J.: Sources and sinks of acetone, methanol, 
and acetaldehyde in North Atlantic marine air, Atmos. Chem. Phys., 5, 1963-1974, 2005,

http://www.atmos-chem-phys.net/5/1963/2005/.

Martin, R. V., Jacob, D. J., Chance, K., Kurosu, T. P., Palmer, P. I., and Evans, M. J.: Global inventory of nitrogen oxide emissions constrained by space-based observations of $\mathrm{NO}_{2}$ columns, J. Geophys. Res., 108(D17), 4537, doi:10.1029/2003JD003453, 2003.

Martin, R. V., Chance, K., Jacob, D. J., Kurosu, T. P., Spurr, R. J. D., Bucsela, E., Gleason, J. F., Palmer, P. I., Bey, I., Fiore, A. M., Li, Q. B., Yantosca, R. M., and Koelemeijer, R. B. A.: An improved retrieval of tropospheric nitrogen dioxide from gome, J. Geophys. Res.-Atmos., 107, 4437, doi:10.1029/2001JD001027, 2002.

Martinez, M., Harder, H., Kubistin, D., Rudolf, M., Bozem, H., Eerdekens, G., Fischer, H., Gurk, C., Klüpfel, T., Königstedt, R., Parchatka, U., Schiller, C. L., Stickler, A., Williams, J., and Lelieveld, J.: Hydroxyl radicals in the tropical troposphere over the Suriname rainforest: airborne measurements, Atmos. Chem. Phys. Discuss., 8, 15491-15536, 2008,

http://www.atmos-chem-phys-discuss.net/8/15491/2008/.

Matsuda, K., Watanabe, I., Wingpud, V., Theramongkol, P., and Ohizumi, T.: Deposition velocity of $\mathrm{O}_{3}$ and $\mathrm{SO}_{2}$ in the dry and wet season above a tropical forest in northern Thailand, Atmos. Environ., 40, 7557-7564, 2006.

McGregor, G. R. and Nieuwolt, S.: Tropical climatology: an introduction to the climates of the low latitudes (Second Edition), John Wiley \& Sons, Chichester, 339 pp., 1998.

Misztal, P. K., Nemitz, E., Langford, B., Coyle, M., Ryder, J., DiMarco, C., Phillips, G., Oram, D., Owen, S., and Cape, J. N.: First direct ecosystem fluxes of VOCs from oil palms in SE Asia, Atmos. Chem. Phys. Discuss., in preparation, 2010a.

Misztal, P. K., Owen, S., Guenther, A., Geron, C., Rasmussen, C., Phillips, G., Ryan, A., Edwards, D. P., Hewitt, C. N., Nemitz, E., and Cape, J. N.: Large estragole fluxes from oil palms in Borneo, Atmos. Chem. Phys. Discuss., submitted, 2010b.

Moura-Costa, P. H., Yap, S. W., Ong, C. L., Ganing, A., Nussbaum, R., and Mojiun, T.: Large scale enrichment planting with dipterocarps as an alternative for carbon offset - methods and preliminary results, 1996, in: Proceedings of the Fifth Round Table Conference on Dipterocarps, edited by: Appanah, S. and Khoo, K. C., Chiang Mai, Thailand, November 1994.

MPOC.: Fact sheet, Malaysian Palm Oil Council, Brussels, 2008.

Nemitz, E., Jimenez, J. L., Huffman, J. A., Ulbrich, I. M., Canagaratna, M. R., Worsnop, D. R., and Guenther, A. B.: An eddycovariance system for the measurement of surface/atmosphere exchange fluxes of submicron aerosol chemical species - First application above an urban area, Aerosol Sci. Tech., 42, 636657, 2008.

Nemitz, E., Phillips, G. J., Di Marco, C. F., Misztal, P. K., Farmer, D., Kimmel, J., and Jimenez, J. L.: Concentrations and surface/atmosphere exchange of aerosol chemical components in $\mathrm{PM}_{1}$ above an oil palm plantation in Sabah, Malaysia, Atmos. Chem. Phys. Discuss., in preparation, 2010.

Olson, J.: World ecosystems (WE1.4): Digital raster data on a 10 minute geographic 1080 × 2160 grid. Global Ecosytems Database, Version 1.0: Disc A. N. G. D. Center, Boulder CO, Nat. Ocean. Atmos. Admin., 1992.

Olson, D. M., Dinerstein, E., Wikramanayake, E. D., Burgess, N.
D., Powell, G. V. N., Underwood, E. C., D’Amico, J. A., Itoua, I., Strand, H. E., Morrison, J. C., Loucks, C. J., Allnutt, T. F., Ricketts, T. H., Kura, Y., Lamoreux, J. F., Wettengel, W. W., Hedao, P., and Kassem, K. R.: Terrestrial Ecoregions of the World: A New Map of Life on Earth, BioScience, 51(11), 933-938. 2001.

Olson, J. R., Crawford, J. H., Chen, G., Brune, W. H., Faloona, I. C., Tan, D., Harder, H., and Martinez, M.: A reevaluation of airborne Hox observations from Nasa field campaigns, J. Geophys. Res.Atmos., 111, D10301, doi:10.1029/2005JD006617, 2006.

Osborne, S. R., Johnson, B. T., Haywood, J. M., Baran, A. J., Harrison, M. A. J., and McConnell, C. L.: Physical and optical properties of mineral dust aerosol during the Dust and Biomass-burning Experiment, J. Geophys. Res.-Atmos., 113(14), D00C03, doi:10.1029/2007JD009551, 2008.

Palmer, P. I., Jacob, D. J., Fiore, A. M., Martin, R. V., Chance, K., and Kurosu, T. P.: Mapping isoprene emissions over North America using formaldehyde column observations from space, J. Geophys. Res., 108(D6), 4180, doi:10.1029/2002JD002153, 2003.

Pearson, G., Davies, F., and Collier, C. G.: An analysis of the performance of the UFAM pulsed Doppler LIDAR for observing the boundary layer, J. Atmos. Ocean. Tech., 26, 240-250, 2009.

Pearson, G., Davies, F., and Collier, C. G.: Remote sensing of the tropical rainforest boundary layer using pulsed Doppler lidar, Atmos. Chem. Phys., in preparation, 2010.

Penkett, S. A., Bandy, B. J., Reeves, C. E., McKenna, D., and Hignett, P.: Measurements of peroxides in the atmosphere and their relevance to the understanding of global tropospheric chemistry, paper presented at Faraday Discussions on Atmospheric Chemistry - Measurements, Mechanics and Models, Royal Soc Chemistry, E Anglia, England, 19-21 April, 1995.

Pike, R. C., Lee, J. D., Young, P. J., Moller, S., Carver, G. D., Yang, X., Misztal, P., Langford, B., Stewart, D., Reeves, C. E., Hewitt, C. N., and Pyle, J. A.: Can a global model chemical mechanism reproduce $\mathrm{NO}, \mathrm{NO}_{2}$, and $\mathrm{O}_{3}$ measurements above a tropical rainforest?, Atmos. Chem. Phys. Discuss., 9, 27611-27648, 2009, http://www.atmos-chem-phys-discuss.net/9/27611/2009/.

Pilegaard, K.: Air-soil exchange of $\mathrm{NO}, \mathrm{NO}_{2}$ and $\mathrm{O}_{3}$ in forests, Water Air Soil Pollut., 1, 79-88, 2001.

Pilegaard, K., Skiba, U., Ambus, P., Beier, C., Brüggemann, N., Butterbach-Bahl, K., Dick, J., Dorsey, J., Duyzer, J., Gallagher, M., Gasche, R., Horvath, L., Kitzler, B., Leip, A., Pihlatie, M. K., Rosenkranz, P., Seufert, G., Vesala, T., Westrate, H., and Zechmeister-Boltenstern, S.: Factors controlling regional differences in forest soil emission of nitrogen oxides ( $\mathrm{NO}$ and $\mathrm{N}_{2} \mathrm{O}$ ), Biogeosciences, 3, 651-661, 2006,

http://www.biogeosciences.net/3/651/2006/.

Plane, J. M. C. and Saiz-Lopez, A.: Analytical Techniques for Atmospheric Measurement, in: Analytical Techniques for Atmospheric Measurement, edited by: Heard, D. E., Blackwell, Oxford, 2006.

Pugh, T. A. M., MacKenzie, A. R., and Hewitt, C. N.: Modelling of nighttime near-surface chemistry in a South-East Asian tropical rainforest, Atmos. Chem. Phys., in preparation, 2010a.

Pugh, T. A. M., MacKenzie, A. R., Hewitt, C. N., Langford, B., Edwards, P. M., Furneaux, K. L., Heard, D. E., Hopkins, J. R., Jones, C. E., Karunaharan, A., Lee, J., Mills, G., Misztal, P., Moller, S., Monks, P. S., and Whalley, L. K.: Simulating atmospheric composition over a South-East Asian tropical rainforest: 
performance of a chemistry box model, Atmos. Chem. Phys. Discuss., accepted, $2010 \mathrm{~b}$.

Redelsperger, J.-L., Thorncroft, C. D., Diedhiou, A., Lebel, T., Parker, D. J., and Polcher, J.: African Monsoon Multidisciplinary Analysis: An International Research Project and Field Campaign, B. Am. Meteor. Soc., 87, 1739-1746, 2006.

Reeves, C. E., Slemr, J., Oram, D. E., Worton, D., Penkett, S. A., Stewart, D. J., Purvis, R., Watson, N., Hopkins, J., Lewis, A., Methven, J., Blake, D. R., and Atlas, E.: Alkyl nitrates in outflow from North America over the North Atlantic during Intercontinental Transport of Ozone and Precursors 2004, J. Geophys. Res.-Atmos., 112(21), D10S37, doi:10.1029/2006JD007567, 2007.

Roberts, G. C. and Nenes, A.: A continuous-flow streamwise thermal-gradient $\mathrm{CCN}$ chamber for atmospheric measurements, Aerosol Sci. Tech., 39, 206-221, 2005.

Ryder, J., Langford, B., Oram, D., Coyle, M., Phillips, G., Helfter, C., Misztal, P., Cape, N., and Nemitz, E.: Sources and sinks of BVOCs inside a SE Asian rainforest canopy, Atmos. Chem. Phys. Discuss., in preparation, 2010.

Saunders, S. M., Jenkin, M. E., Derwent, R. G., and Pilling, M. J.: Protocol for the development of the Master Chemical Mechanism, MCM v3 (Part A): tropospheric degradation of nonaromatic volatile organic compounds, Atmos. Chem. Phys., 3, 161-180, 2003,

http://www.atmos-chem-phys.net/3/161/2003/.

Saunois, M., Reeves, C. E., Mari, C. H., Murphy, J. G., Stewart, D. J., Mills, G. P., Oram, D. E., and Purvis, R. M.: Factors controlling the distribution of ozone in the West African lower troposphere during the AMMA (African Monsoon Multidisciplinary Analysis) wet season campaign, Atmos. Chem. Phys., 9, 61356155,2009 ,

http://www.atmos-chem-phys.net/9/6135/2009/.

Schmitt, C., Belokurov, A., Besançon, C., Boisrobert, L., Burgess, N.D., Campbell, A., Coad, L. Fish, L., Gliddon, D., Humphries, K., Kapos, V., Loucks, C., Lysenko, I., Miles, L., Mills, C., Minnemeyer, S., Pistorius, T., Ravilious, C., and Winkel, G.: Global Ecological Forest Classification and Forest Protected Area Gap Analysis: Analyses and Recommendations in view of the $10 \%$ target for forest protection under the Convention on Biological Diversity (CBD). Freiburg University Press, Freiburg, Germany, 2008.

Serca, D., Guenther, A., Klinger, L., Vierling, L., Harley, P., Druilhet, A., Greenberg, J., Baker, B., Baugh, W., Bouka-Biona, C., and Loemba-Ndembi, J.: Expresso flux measurements at upland and lowland Congo tropical forest site, Tellus B, 53, 220-234, 2001.

Stewart, D. J., Taylor, C. M., Reeves, C. E., and McQuaid, J. B.: Biogenic nitrogen oxide emissions from soils: impact on NOx and ozone over west Africa during AMMA (African Monsoon Multidisciplinary Analysis): observational study, Atmos. Chem. Phys., 8, 2285-2297, 2008, http://www.atmos-chem-phys.net/8/2285/2008/.

Still, T. J., Al-Haider, S., Seakins, P. W., Sommariva, R., Stanton, J. C., Mills, G., and Penkett, S. A.: Ambient formaldehyde measurements made at a remote marine boundary layer site during the NAMBLEX campaign - a comparison of data from chromatographic and modified Hantzsch techniques, Atmos. Chem. Phys., 6, 2711-2726, 2006, http://www.atmos-chem-phys.net/6/2711/2006/.

Tan, D., Faloona, I., Simpas, J. B., Brune, W., Shepson, P. B., Couch, T. L., Sumner, A. L., Carroll, M. A., Thronberry, T., Apel, E., Riemer, D., and Stockwell, W.: HOx budgets in a deciduous forest: Results from the PROPHET summer $1998 \mathrm{cam}$ paign, J. Geophys. Res.-Atmos., 106(D20), 24407-24427, 2001.

Tangki, H.: Biomass variation across selectively logged forest in Borneo and its prediction by Landsat TM data, Unpublished MPhil thesis, Lancaster University, UK, 2008.

Tangki, H., and Chappell, N. A.: Biomass variation across selectively logged forest within a $225 \mathrm{~km}^{2}$ region of Borneo and its prediction by Landsat TM, Forest Ecol. Manage., 256, 19601970, 2008.

Thomas, R. M., Trebs, I., Otjes, R., Jongejan, P. A. C., Ten Brink, H., Phillips, G., Kortner, M., Meixner, F. X., and Nemitz, E.: An Automated Analyzer to Measure Surface-Atmosphere Exchange Fluxes of Water Soluble Inorganic Aerosol Compounds and Reactive Trace Gases, Environ. Sci. Technol., 43, 1412-1418, 2009.

UNEP-WCMC: Global Distribution of Original and Remaining Forests, United Nations Environment Programme - World Conservation Monitoring Centre (UNEP-WCMC), online available at: http://www.unep-wcmc.org/forest/original.htm, 2000.

Voulgarakis, A., Savage, N. H., Wild, O., Carver, G. D., Clemitshaw, K. C., and Pyle, J. A.: Upgrading photolysis in the pTOMCAT CTM: model evaluation and assessment of the role of clouds, Geosci. Model Dev., online available at: www. geosci-model-dev.net/2/59/2009/2, 59-72, 2009.

Voulgarakis, A., Savage, N. H., Braesicke, P., Wild, O., Carver, G. D., and Pyle, J. A.: Interannual variability of tropospheric composition: the influence of changes in emissions, meteorology and clouds, Atmos. Chem. Phys. Discuss., 9, 14023-14057, 2009, http://www.atmos-chem-phys-discuss.net/9/14023/2009/.

Volz-Thomas, A., Lerner, A., Patz, H. S., Schultz, M., McKenna, D., Schmitt, R., Madronich, S., and Roth, E.: Airborne Measurements of the Photolysis of $\mathrm{NO}_{2}$, J. Geophys. Res.-Atmos., 101, 18613-18627, 1996.

Walsh, R. P. D.: Climate. The Tropical Rain Forest, edited by: Richards, P. W., 159-205, Cambridge University Press, Cambridge, 352 pp., 1996.

Whalley, L. K., Furneaux, K. L., Goddard, A., Lee, J. D., Mahajan, A., Oetjen, H., Read, K. A., Carpenter, L. J., Lewis, A. C., Plane, J. M. C., Saltzman, E. S., and Heard, D. E.: The Chemistry of $\mathrm{OH}$ and $\mathrm{HO}_{2}$ in the Boundary Layer over the tropical Atlantic Ocean, Atmos. Chem. Phys. Discuss., in preparation, 2010a.

Whalley, L. K., Furneaux, K. L., Heard, D. E.: Measurements of hydroxyl radicals over a SE Asian tropical rainforest, Atmos. Chem. Phys Discuss., in preparation, 2010b.

Whalley, L. K., Lewis, A. C., McQuaid, J. B., Purvis, R. M., Lee, J. D., Stemmler, K., Zellweger, C. and Ridgeon, P.: Two highspeed, portable GC systems designed for the measurement of non-methane hydrocarbons and PAN: Results from the Jungfraujoch High Altitude Observatory, J. Environ. Monit., 6, 234-241, 2004.

Whitmore, T. C.: Tropical rain forests of the Far East, 2nd edition, Oxford University Press, Oxford, 1984.

Whitmore, T. C.: An introduction to tropical rain forests, 2nd edition, Clarendon, Oxford, 1998.

Wild, O., Law, K. S., McKenna, D. S., Bandy, B. J., Penkett, S. A., and Pyle, J. A.: Photochemical trajectory modeling studies of 
the North Atlantic region during August 1993, J. Geophys. Res., 101, 29269-29288, 1996.

Wilkinson, M. J., Owen, S. M., Possell, M., Hartwell, J., Gould, P., Hall, A., Vickers, C., and Hewitt, C. N.: Circadian control of isoprene emissions from oil palm (Elaeis guineensis), The Plant Journal, 47, 960-968, doi:10.1111/j.1365-313X.2006.02847.x, 2006.
Wolter, K. and Timlin, M. S.: Measuring the strength of ENSO how does 1997/98 rank?, Weather, 53, 315-324, 1998.

Worton, D. R., Mills, G. P., Oram, D. E., and Sturges, W. T.: Gas chromatography negative ion chemical ionization mass spectrometry: Application to the detection of alkyl nitrates and halocarbons in the atmosphere, J. Chromatogr. A, 1201, 112-119, 2008. 